\title{
Coalitionally Stable Pricing Schemes for Inter-domain Forwarding
}

\author{
Onkar Bhardwaj*, Elliot Anshelevich, Koushik Kar \\ Rensselaer Polytechnic Institute, Troy, NY 12180.
}

\begin{abstract}
In this work, we model and analyze the problem of stable and efficient pricing for inter-domain traffic routing in the future Internet. We consider a general network topology with multiple sources and sinks of traffic, organized into separate domains managed by Internet Service Providers (ISPs) solely interested in maximizing their own profit. In this framework, we prove that there exists a pricing scheme that attains network-wide efficiency and is yet coalitionally stable, where the coalitions correspond to the ISPs that are acting in self-interest. This implies that this pricing scheme not only maximizes the overall utility of the resulting traffic flows, but is also such that ISPs cannot expect to improve their profit through deviation from it, even if multiple ISPs deviate at the same time. Through simulations on scale-free preferential attachment network topology models as well as actual inter-domain topologies obtained from the CAIDA database, we evaluate the convergence of best-response based simple price updates, and show that they quickly attain near-optimal network utility in these network topologies.
\end{abstract}

Keywords: Inter-domain forwarding, Traffic pricing, Coalitional stability

\section{Introduction}

\subsection{Background and Motivation}

Current inter-domain traffic routing and pricing practices are typically driven by simple heuristic rules and policies, and do not in general optimize the overall use of Internet resources. Inter-domain routing follows the Border Gateway Protocol (BGP) standard [42] that only attempts to minimize the number of ISP hops on the path of a flow, in addition to following local policy considerations and certain heuristic rules. Traffic exchange contracts between ISPs are mostly negotiated manually and span months, even years. Even at these long timescales, these contracts are often simplistic, with no detailed quality metrics, and usually specifying a minimum base traffic volume for charging. Charging is typically done based on the total traffic offered by the customer ISP, irrespective of their destination; this is also refereed to as 'point-to-anywhere pricing' [46]. It has been argued correctly that point-to-point or destination-based pricing can result in better efficiency of network resource usage [52]. Moreover, recent measurements also show fast-changing patterns for interdomain traffic [31]; inter-domain service pricing and traffic engineering solutions need to be flexible enough to adapt to such dynamically changing traffic patterns, as well as variations in customer requirements and their willingness to pay.

* Corresponding author

Email addresses: onkar.bhardwaj@gmail.com, +1-603-369-1125 (Onkar Bhardwaj), eanshel@cs.rpi.edu (Elliot Anshelevich), koushik@ecse.rpi.edu (Koushik Kar) 
High-efficiency inter-domain traffic engineering involves many challenges ranging from technical capabilities to economics and policy [16]. One of the difficulties is that it requires involvement of (agreement by) multiple Internet Service Providers (ISPs) that are naturally interested in maximizing their own profits, and their individual objectives are often in conflict with each other. While multi-ownership is in general essential for competition, efficiency, and growth of a system, the multi-provider structure of the Internet has been a hindrance towards attaining efficiency in routing beyond individual ISP or Autonomous Systems (AS) boundaries. These facts also imply that an inter-domain traffic engineering solution will only be adopted easily if it allows individual ISP business goals and policies to be respected, and is economically profitable/justifiable to each ISP. Another challenge in this context is the counter-productive effects of intra-domain and inter-domain traffic engineering policies if they are not designed with careful consideration of their interaction with each other $[24,5,2]$.

Economically driven inter-domain traffic engineering is a challenging task, and in this work we undertake some initial steps towards studying this complex yet important issue. We provide and analyze a game-theoretic model of price-setting by ISPs that are solely interested in maximizing their individual profits, taking into account the changes to the traffic pattern resulting from this price change. We study the question whether a "good" equilibrium exists, i.e, whether there exists a price-setting solution from which profit-maximizing ISPs would not deviate from (out of selfinterest), and yet results in a traffic flow that maximizes the overall network utility (social welfare).

\subsection{Focus of this Work}

The pricing and forwarding questions for inter-domain traffic are intertwined: the "price of forwarding" as chosen by an ISP will naturally impact the traffic it receives, as it will determine how much traffic its neighboring ISPs will offer. Similarly, traffic routing by an ISP from the ingress to the egress nodes through its internal network will depend on the prices offered by its neighboring nodes, since each ISP would naturally like to send flow to the destination at minimum cost. The question we study in this paper involves finding the "right" price that ISPs should charge for traffic forwarding services within the framework of destination-based, next-hop routing, which is the current Internet practice. Note that an ISP obtains payments from its upstream neighbors for accepting their traffic for forwarding, and must in turn pay its downstream neighbors for receiving its traffic (i.e., the traffic it has accepted to forward, plus the traffic that originates within its own network).

We assume that ISPs are interested in maximizing their individual profits, defined as the revenue (or utility) an ISP earns by accepting its neighbors' traffic as well as that of its own subscribers (end-users), minus the cost incurred for sending traffic to its neighbors. In this framework, the question we consider in this paper is how inter-domain traffic forwarding services should be priced so that it is stable and efficient. Posed another way, we address the question of whether efficient inter-domain traffic engineering can be realized within a framework where ISPs compete with each other for traffic and revenue. More specifically, this comprises of studying the two following issues: (i) The existence of equilibrium prices, from which ISPs (with the ability to anticipate how the traffic would vary in response to price changes) would not have any incentive to deviate; (ii) The efficiency of this pricing equilibrium, in terms of maximizing the overall network utility (generalized throughput) defined solely in terms of the traffic flows.

Since we pose our question as a complete information game, our model implicitly assumes that ISPs can correctly anticipate (or estimate) the effect of their price changes on the traffic patterns. 
Computing such an equilibrium would in general require global knowledge, and is therefore infeasible in practice. Therefore we also explore the convergence of an incremental best-response based price update policy that is motivated by price update/discovery mechanism used in a typical market-place. This incremental price update strategy can be implemented without knowledge of the network, the impact of the price change being inferred simply by observing the changed behavior of the flows reacting to the price change (the price update is assumed to occur in a relatively slow timescale for this to happen).

\subsection{Contribution and Significance}

We provide a game-theoretic model of price-setting by ISPs, where each ISP is solely interested in maximizing its profits, while anticipating (or estimating) the changes to the overall traffic pattern that will result from changes to its pricing scheme. We establish the existence and efficiency of a coalitional equilibrium for this game, where each coalition corresponds to an ISP network. In our model, an ISP is represented as a cloud - a subnetwork of nodes (routers) and edges (links) connected in an arbitrary topology, or even a collection of (possibly disjoint) subnetworks. This general ISP model is well representative of the administrative structure in the current Internet where an ISP can control multiple Autonomous Systems (ASes) [57], and is much more general than models which assume that each ISP consists only of a single node in the network (see Section 2). Our view of ISPs as coalitions aligns well with the fact that an ISP will jointly optimize the prices it quotes at all its ingress points, along with the traffic forwarding paths inside its network.

The concept of stability (coalitional equilibrium) considered by us is substantially strong: It is resilient to (i) ISPs being optimistic about the flows that may result from changing their price; (ii) any ownership (coalition) structure in the network, or equivalently, any partitioning of the network (Internet) into ISP boundaries. The notion of social optimality we consider amounts to maximizing the aggregate utility (generalized network throughput) in the entire network (Internet); and this does not need to take into account the ISP boundaries. Note that our notions of coalitional equlibrium and coalitional stability are also alternatively known in the literature as strong Nash equilibrium and strong stability (of Nash equilibria), respectively.

Despite our strong stability concept, we show that socially optimal flows are stabilizable, i.e., we show how to compute prices which together with a socially optimal flow lead to a coalitional equilibrium. In other words, we show that the price-of-stability (PoS) is unity: there are stable solutions which are as good as the social optimum, and so we do not need to sacrifice any social welfare in order to obtain stable prices. This holds not only when per-node traffic constraints are modeled as a hard capacity limit but also when they are modeled as convex transit costs. These results can be shown to extend even if capacities or transit costs are associated with links instead of nodes. The corresponding prices would not only maximize the total utility of the flow resulting in the Internet, but will remain stable even if the ownership structures (ISP boundaries) change, as long as the underlying physical topology remains unaltered.

It is important to note that our work is different from the classic work where similar pricing schemes have been shown to result in a Walrasian (a.k.a., competitive) equilibrium for several network contexts (see e.g., $[29,28]$ ) - which in our context is essentially equivalent to saying that for a socially optimal flow no player has any incentive to reroute their traffic given these pricing schemes. However, unlike these classic results, in our case the players are also in charge of setting the prices for the goods: equilibrium prices for our setting does not only mean that everyone maximizes their utility given these prices, but also that no one could change their prices and obtain 
greater utility. This is important because Walrasian equilibrium per se makes no guarantees on the stability of players who are price-setting, or on the stability of coalitions. In fact, there are many settings where if a player is able to change the price of a good in a Walrasian equilibrium (instead of these prices being exogenously specified), then the players would have great incentive to do so, and would lead to loss of stability. The novelty of our results is that for our setting, we show this coalitional equilibrium to be stable also with respect to the price-setters changing their prices, not just to changing the flow amounts given exogenously specified prices. Additionally, our notion of coalitional stability implies that such an equilibrium is stable even with respect to fixed or dynamic coalitions in the network, i.e., the equilibrium is stable not only with respect to individual ISP clouds but also with respect to arbitrary subnetworks forming coalitions, several ISP's merging into one, etc.

Another important point to note is that unlike most of the existing related work (as we discuss in the next section) our results apply to any number of sources and destinations of traffic, and to ISPs which consist of an entire network, instead of a single node. Through simulations on random network topologies as well as scale-free preferential attachment based network topologies, we evaluate the convergence of a best-response based simple price update solution where an ISP changes its price incrementally if the change improves its profit. We observe that this incremental price update process converges quickly in all cases simulated, and show that it attains near-optimal network utility in the majority of cases, for both the popularly used scale-free preferential attachment network topology generation model, as well as a real AS-level topology obtained from the CAIDA database.

To conclude our discussion on the significance of our results, a final note on the applicability of our model and its limitations is in order. Firstly, our traffic pricing-forwarding game model does not adequately represent the inter-domain routing and pricing/contracting practices in the current Internet, where ISPs pay each each other for the connectivity (to destination network prefixes) provided by the other party, and not on the basis of the units of flow forwarded on a per-destination basis. Furthermore, an important restriction in our model is that the payments are directional, and only one end-point of the communication pair receives utility from this communication. This is not true for the current Internet either, where it can be argued that both end-points of a communication may receive benefit from it. Furthermore, payments for traffic exchange over the Internet are not unidirectional: payment flows "uphill" from the end-customers towards core ISPs on both sides, meeting at a common ISP or two peering ISPs [18]. Therefore, in general both end-points share the cost of communication (connectivity) in the current Internet. While the extension of our results to current Internet practices still remains open, we believe that our framework is well-aligned with some of the future Internet inter-domain routing structures $[20,10,52]$ that propose more flexible routing/contracting on a flatter (less hierarchical) inter-domain structure. Unidirectional flow of payments fit more naturally in these frameworks. The other motivation of our model can be found by considering the growth of 'cloud computing' in the Internet, where the cloud servers are often located "uphill" (towards the Internet core) from the client. The cost of traffic forwarding through the Internet can either be borne by the client which derives direct utility from this communication; alternatively, the cloud server could also make the payments for this traffic exchange, although that cost may be accounted for in the service contract between the cloud service provider and the client. Mapped to our model, the two end-points of communication would be the client and server associated with the cloud service, and the directionality of the payments (from the one end-point that receives direct utility from the communication, towards the other end-point) could be justified. 


\subsection{Paper Organization}

The rest of the paper is structured as follows. In Section 3, we describe the system model and provide some observations/results on the special case where each ISP is modeled as a single node; these motivate the main results that follow in the next few sections. In Sections 4-5, we state and prove our main results. In Section 6, we show that our results extend when we have transit costs instead of hard capacities at nodes. Section 7 presents our simulation results on a simple best-response based pricing update policy. Finally, we conclude in Section 8 listing possible future directions and outlining the limitations of our work.

\section{Related Work}

Research in inter-domain traffic engineering has gained momentum over the last decade, although the bulk of this work has been devoted to inter-domain traffic engineering solutions in the context of BGP, through intelligent use of some of its parameters/flexibilities. Prior work such as $[40,41]$ discuss methods for doing inter-domain traffic engineering by careful control of BGP route advertisements, while [19] discusses how the BGP AS-Path attribute can be manipulated for that purpose. General guidelines for traffic engineering within the context of BGP are discussed in [16]. The authors in [50] discuss an extension to BGP to enable multipath inter-domain routing. A cooperative optimization-based approach to inter-domain traffic engineering based on dual decomposition and Nash bargaining is described in [47]. The work [48] analyzes the current tiered pricing practices in the wholesale transit market, and suggest that incorporating both traffic demand and carrying cost into a small number of tiers yields good performance. Unlike our work, the above line of work on inter-domain traffic engineering does not take into account strategic pricing by different ISPs/domains or study the inter-domain traffic engineering question from a game-theoretic perspective. The efficiency of selfish flow routing by users is considered in [43] whereas [2] studies flow equilibrium efficiency with optimal routing at the intra-domain level and selfish routing across domains. The pricing competition question across domains (ISPs) is however not considered in these works.

Technically, our work is related to the growing body of literature on strategic pricing of bandwidth/flow/services over the Internet, although most of the existing work uses restrictive network topology models [37]. The flow and price control leader-follower game between a set of users (follower) and an ISP (leader) - which controls a link, or a set of tandem links - is studied in [7, 8]. The authors in $[13,17]$ study the question of pricing/tolling of network edges (links) towards realizing efficient Nash flow equilibria, but these works do not address the issue of price competition between providers. For a single profit-maximizing ISP, equilibrium efficiency questions are studied in [25] whereas [33] considers pricing competition between multiple providers at a single access point, and shows that Nash equilibria are socially efficient; however this work does not consider more general topologies, or coalitional deviations. The work [23] investigates the inefficiency of Nash equilibria in a pricing game model with general concave user utilities, and proposes a revenue-sharing policy that encourages ISPs to cooperate towards attaining better efficiency.

Next-hop routing and pricing models have been considered in, for example, [38] and [49]. However, these papers only consider a single-source single-sink network, and associate latency functions with links instead of link capacities. Next-hop inter-domain routing as in BGP is analyzed in [21], but this work focuses on the issue of incentive-compatibility; [32] looks at the incentive-compatibility of best-response dynamics in BGP routing. [4] considers a somewhat general routing game that can 
also include next-hop routing as a special case, but uses a very different pricing mechanism from the one considered here. Specifically, in our paper the ISP can change the price that it is asking to forward traffic unilaterally, while in [4] a price is considered to be a bilateral contract, so that it takes both participants (the payer and the payee) in order to change the asking price.

Our PATH model can be interpreted in classic economic theory as an instance of an exchange economy: transit nodes are selling bandwidth, and source nodes are purchasing it. The study of such markets, and the Walrasian (a.k.a. competitive) equilibria in these settings, is a major area in Economics, see for example [35]. However, unlike these classic results, in our case the players are also in charge of setting the prices for the goods: equilibrium prices for our setting does not only mean that everyone maximizes their utility given these prices, but also that no one could change their prices and obtain greater utility. In fact, one way of interpreting our result that there exist stable prices that bring about the optimum flow is: "In this context, a Walrasian equilibrium is also stable with respect to coalitional deviations of firms simultaneously changing their prices."

Our coalitional solution concept should not be confused with concepts such as the core in cooperative games. When a coalition of ISPs decide to change their prices, they still remain a part of the overall network, and receive flow from outside the coalition. When studying the core for routing games (see for example Markakis et al. [34]), coalitions can secede from the system entirely if it would benefit them. There are many other differences between our work and that of papers such as [34]: for example, [34] does not consider prices, and the actual goal of all the players is to maximize the flow leaving it. In our case, the goal of a transit node is to maximize its profit; it does not care about the flow being routed except in the sense that it makes money from it.

Because in our setting the players are in control of the prices, and may change them if it is to their benefit, this work is also relevant to the area of path auctions, and in general settings where firms or edges can choose prices while anticipating their return based on these prices. In path auctions (e.g. [27]), edges choose prices, and the cheapest path is purchased; such work usually only involves a single path and integral purchase of edges. In papers such as $[38,3,36,22]$, the edges in the network choose prices, and the cost of a route depends both on these prices and on the latency cost of this route. Thus, instead of choosing the cheapest route, source nodes also must worry about the routes of others, and the resulting latency, resulting in a Nash equilibrium of a congestion game. This setting considers ISPs to be single nodes instead of subnetworks, does not include edge capacities, and is usually analyzed only for special network types such as parallel links or parallel-path networks. While inter-domain pricing-forwarding games have been considered in our recent work as well [1], the results there have been obtained under a restricted model in which there is a single destination node, the internal capacities of ISPs are sufficiently large, and certain link capacity assumptions are satisfied to ensure that the system remains non-monopolistic. Not only is the analysis technique of the current paper substantially different from [1], we also obtain much stronger results that apply to multiple destinations without strong assumptions on the network topology to ensure non-monopolistic behavior. Furthermore, our results apply to "network of networks", i.e., when the intra-ISP topologies are accounted for as well; however, it can be shown that the model in [1] may not even admit Nash equilibrium unless strong assumptions on the intra-ISP topologies are made.

The authors in $[14,30]$ use a very similar model to our PATH model with convex costs. Although they only consider single-node ISP topologies (i.e., each node is operated by an independent entity), another major difference which completely changes the equilibrium properties is that in $[14,30]$ each player is only allowed to choose quadratic pricing functions (i.e., the price grows linearly with the 


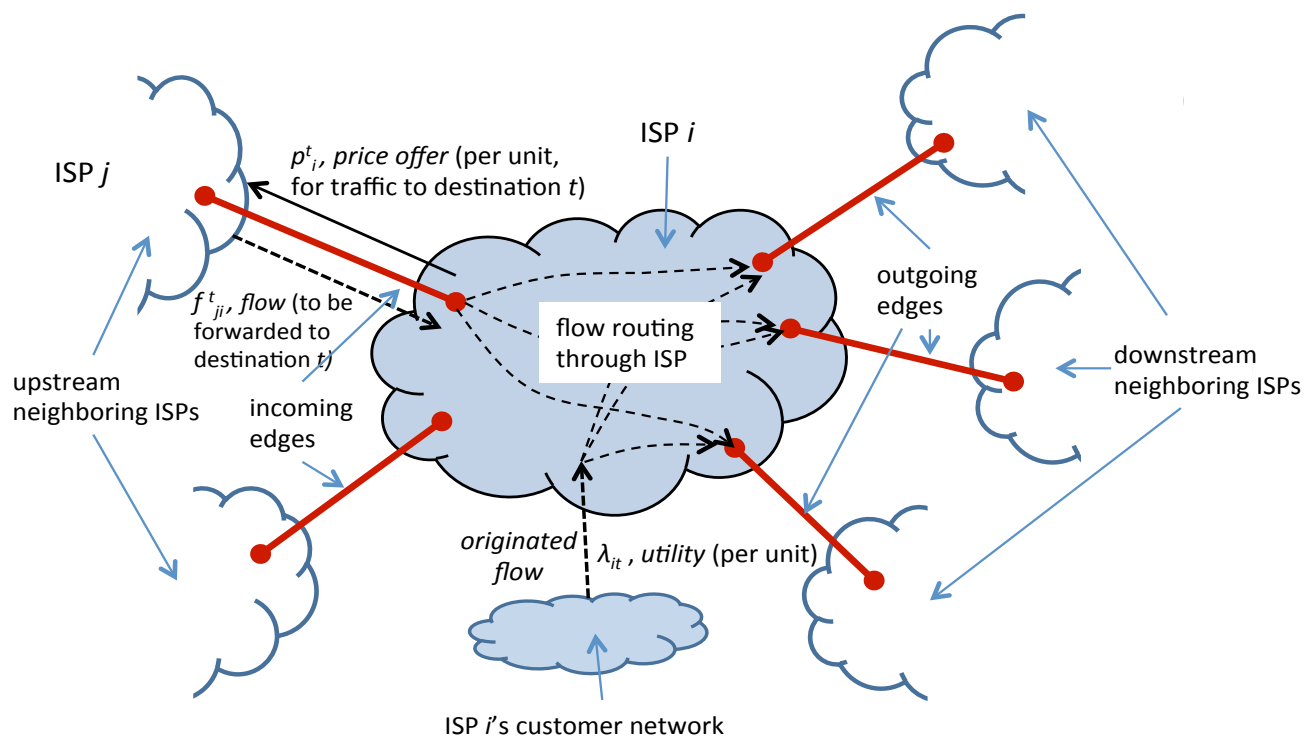

Figure 1: Price setting, flow generation and forwarding by an ISP $i$ for traffic to a destination $t$.

amount of load), while in our model each player chooses linear price functions (i.e., a constant price for each unit of bandwidth). Both types of price functions are reasonable for different contexts. We would argue that having linear pricing is more natural and common, and leads to simpler user behavior: users simply choose routes that cost the least, instead of expecting the cost of the route to change based on the choices made by other users. Finally, $[12,11]$ consider a very similar model to our PATH model. However, $[12,11]$ do not consider players that may control an entire subnetwork, and establish mostly negative results on the nature of the equilibrium for the case of multiple destinations. While we specifically consider linear source utilities $([12,11]$ consider more general source utilities), we show the existence of stable pricing for optimal flows, even when players can control more than a single node each, i.e., each player can control a subnetwork, representing the internal network of an ISP (player).

\section{Model and Equivalence Results}

We consider the multi-provider structure of the Internet by modeling it as a "network of networks", where each ISP is a network (subnetwork), or a collection of networks (subnetworks). The ISPs quote destination-specific prices to each other for forwarding/transiting the traffic for a specific destination, on the inter-ISP links that connect them. It is also through these inter-domain links that ISPs direct flows toward each other, which are directed through the ISP's internal network topology, possibly to other neighboring ISPs to the destination. Other than forwarding each other's traffic, ISPs also generate and forward traffic of their own, which originate from the end-users that the ISP directly serves. Prices are quoted by the ISPs, and traffic is generated and forwarded by them (in response to the prices quoted by their neighbors), so that their individual profits are maximized. Figure 1 overviews the pricing and forwarding scheme from the perspective of ISP $i$.

For simplicity of exposition of the basic ideas, the discussion in the rest of this section assumes that each ISP is abstracted (modeled) as a single node. In this case, the internal topology of an ISP becomes inconsequential, and gets represented as a nodal capacity constraint. Our main 
results - those for the general model where each ISP is modeled as a subnetwork (or collection of subnetworks) - are presented in Sections 4 and 5.

\subsection{Prices and Profits}

Next we proceed to describe the pricing scheme that we consider, and how flows are determined in response to those prices; we refer to this as the PRICE ModeL.

We model the network as a graph $G=(V, E)$, where $V$ is the set of nodes (ISPs) and $E$ is the set of directed edges (inter-ISP links), with each node (ISP) in the network being a player. Each node in the network is either a source node or a transit node. As it will be evident from the discussion below, a destination node can be viewed as a special case of a transit node. A source node $s$ desires to send an amount $d_{s t}$ of traffic to some destination node $t$ (different source nodes may want to send traffic to different destinations) and is not willing to transit any traffic of others, while a transit node does not send any traffic of its own, but instead forwards traffic of others. As we will see later, this distinction does not matter, and all our results hold if the same node acts as both a source node and a transit node, or desires to send traffic to multiple destinations; however, it simplifies the exposition to consider source nodes and transit nodes to be separate.

Each transit node $v$ has a capacity $c_{v} \geq 0$ for all traffic being transited through it. While we associate capacities with nodes for simplicity of exposition, our model and results easily extend to the case where capacities are associated with the edges (links) instead. Each source node $s$ can send traffic to destination $t$ up to a maximum rate of $d_{s t}$, and derives a value of $\lambda_{s t} \geq 0$ per-unit of traffic that it sends to destination $t$, i.e. the utility source $s$ derives from sending $x \leq d_{s t}$ traffic to destination $t$ is $\lambda_{s t} \cdot x$.

Each node $v$ 's strategy is to choose a desired profit $\pi_{v} \geq 0$. The profit $\pi_{v}$ specifies how much profit $v$ is determined to make from each unit of traffic that it transits (if $v$ is a transit node), or from each unit of traffic that it sends (if $v$ is a source node). The strategies chosen by the nodes give rise to prices and flows of traffic as follows.

Denote by $p_{v}^{t}$ the price that node $v$ charges for forwarding a unit of traffic to destination $t$. We assume that any node with an edge to $v$ can send any amount $x$ to $t$ through $v$ by paying $p_{v}^{t} \cdot x$. Then, the prices are determined by $p_{v}^{t}=\pi_{v}+\min _{(v, w) \in E} p_{w}^{t}$. In other words, node $v$ looks at the cheapest price that it has to pay to get traffic to $t$, adds its desired profit $\pi_{v}$ to this price, and asks everyone to pay this amount. Thus, the prices are completely determined by the desired profits $\pi_{v}$. Note that the capacity constraints are taken into account when setting prices, albeit implicitly. If a transit node is receiving too much flow, it will raise its price in order to reduce the amount of flow it gets.

Let $p(\pi)$ be the price vector resulting from the profits using the process described above. Note that these prices may differ across destinations, since forwarding to some destinations may be more costly than to other destinations. Given these prices, the source nodes send their flow to their neighbor with the cheapest price (as long as by doing so they receive more than $\pi_{s}$ profit), and transit nodes forward their traffic to their neighbors with the cheapest price. Due to tiebreaking (which can be arbitrary) between several nodes with the same price, many possible flows may form: to make this precise we define the set of reasonable flows as follows.

Remark 1: For ease of exposition, we have assumed directional demands (in which some "source" nodes want to send traffic to some "destination" nodes). However, for our results to hold this does not have to be the case. All our results hold if traffic can flow in any direction between the two communication end-points; what is necessary is that the payments are directional, 
as discussed in Section 1.3. In other words, a "source" node $s$ in our model can be interpreted as a source of payments and a corresponding "destination" node $t$ can be interpreted as a destination for payments for the traffic contributed by the pair $(s t)$ - and the actual physical traffic can flow in any direction between these two, with the source node $s$ receiving a utility of $\lambda_{s t} \cdot x$ for $x$ amount of traffic contributed by the pair $(s t)$.

Definition 1 (Valid flow for PRICE MODEL). A valid flow $f$ given prices $p(\pi)$ satisfies the following properties:

1. Each node $v$ (either a source or a transit node) which sends or forwards flow destined for $t$ forwards it only to neighbors $w$ with the smallest price $p_{w}^{t}$.

2. Let $q=\min _{(s, w) \in E} p_{w}^{t}$ be the minimum price asked by a neighbor of a source node $s$ for forwarding traffic to destination $t$. If $\lambda_{s t}-q>\pi_{s}$, then s sends all of its $d_{\text {st }}$ traffic (since it receives at least the desired $\pi_{s}$ profit for each). If $\lambda_{s t}-q<\pi_{s}$, then it sends none of its traffic. (If $\lambda_{s t}-q=\pi_{s}$, then any amount s sends is allowed.)

In other words, a valid flow is what we would expect to happen when nodes send their traffic to neighbors charging the smallest price, and when sources send traffic only if they would make enough profit. Note that in Definition 1 when $\lambda_{s t}-p_{w}^{t}=\pi_{s}$, the source node $s$ can send any arbitrary amount of traffic and all the resulting flows will fall into the category of valid flow.

We define the utility of a source node $s$ to simply be $U_{s}(\pi, f)=\lambda_{s t} \cdot f_{s t}-p_{w}^{t} \cdot f_{s t}$, where $f_{s t}$ is how much flow node $s$ sends to $t$, and among all the neighbors of $s$, node $w$ is the neighbor offering the cheapest price $p_{w}^{t}$ to forward the traffic to destination $t$. Similarly, the utility of a transit node $v$ is the amount of money it receives minus the amount it spends, i.e., $U_{v}(\pi, f)=\sum_{t}\left[p_{v}^{t} \cdot f_{t}^{i n}-p_{w}^{t} \cdot f_{t}^{i n}\right]$, where $f_{t}^{i n}$ is the amount of flow $v$ receives destined for destination $t$, and $w$ is the neighbor of $v$ with minimum $p_{w}^{t}$. If the total flow being transited by $v$ is more than its capacity $c_{v}$, then the utility of $v$ is assumed to be negative infinity. Thus, a valid flow is simply a flow that would form if all nodes chose to forward flow in order to maximize their utility. Let $F(\pi)$ denote the set of valid flows given profits $\pi$ (and thus prices $p(\pi)$ ).

Note that (as in $[12,11]$ ) when sending traffic to $v$, neighbors of $v$ do not care about the capacity of node $v$. One can think of this interaction as follows: if a transit node becomes overcapacitated, then it will be forced to pay penalties to its customers due to the failure to uphold its routing obligations; for customer nodes, these penalties more than compensate for the loss in traffic throughput, and thus it is always in the customer's interest to forward traffic to the lowestpriced downstream node, regardless of available capacity. If node $v$ is running low on capacity it is always free to increase its desired profit $\pi_{v}$ and thus its asking price, thereby reducing the amount of traffic it receives. Thus prices that lead to over-capacitated nodes are never stable, since the over-capacitated node will always have incentive to increase its profit $\pi_{v}$.

We study stable solutions of this game. Specifically, we call a flow and vector of desired profits stable if the following holds.

Definition 2 (Stable solution for PRICE MODEL). A flow-profit pair $(f, \pi)$ is a stable solution if it satisfies the following properties:

1. $f$ is a valid flow with respect to $p(\pi)$. 
2. No node $v$ can change its desired profit $\pi_{v}$ and obtain a higher utility. Formally, let $\pi^{\prime}=$ $\left(\pi_{v}^{\prime}, \pi_{-v}\right)$ be the profit vector which is identical to $\pi$ except for node $v$ changing its desired profit to any $\pi_{v}^{\prime} \neq \pi_{v}$. Then, $U_{v}(\pi, f) \geq U_{v}\left(\pi^{\prime}, f^{\prime}\right)$, for every valid flow $f^{\prime} \in F\left(\pi^{\prime}\right)$.

Thus, no matter what valid flow may form due to node $v$ changing its strategy to $\pi_{v}^{\prime}$, if the solution is stable then node $v$ would not be better off. This definition is stronger than the usual Nash equilibrium: no matter which valid flow would result from changing its strategy to $\pi_{v}^{\prime}$, each node $v$ should still not be able to benefit. Although this solution concept is quite strong, we show that it always exists, and in fact that by choosing appropriate profits $\pi$ we are able to stabilize the optimum flow (i.e., maximize total network utility at equilibrium). In other words, the price of stability is 1: there are stable solutions which are as good as the social optimum, and so we do not need to sacrifice any social welfare in order to obtain stable prices.

\subsection{Equivalence of Price and Path Models}

Since the prices are completely determined by the profits $\pi$ in the above model, we can define the following equivalent model (which we call the PATH MoDEL) by omitting prices entirely as described below. Subsequently, we use this equivalent and intuitively simpler PATH ModeL to prove the main results on the inter-domain pricing-forwarding game that we consider in this paper.

Why define two models?. Before defining the PATH Model, we discuss the differences between the Price and the Path Models, and why we define both in this paper. The Price Model is the one which is most relevant to the routing applications that we consider, and thus we are mostly interested in results for the PrICE MODEL. As described in the previous section, in this model the nodes choose desired profits, which gives rise to prices for transiting flow, which gives rise to a valid flow, which in turns determines the payoff of each node. In the PATH ModeL, the strategies of the players are exactly the same (just choosing desired profits), but there are no prices: instead the flow simply chooses the cheapest path according to the sum of $\pi_{v}$ values. In other words, instead of sending flow locally to the cheapest next-hop price, in the PATH MoDEL the route is determined globally by considering the cheapest overall path. The PATH MODEL is interesting in its own right: consider for example a future Internet routing protocol which actually looks at the cost of routing the traffic for the entire path, instead of locally considering the next-hop price.

But perhaps the most useful thing about the PATH MODEL for our purposes is that, as we show in this section, it is equivalent to the PRICE MODEL, while also being easier to analyze. Therefore, throughout most of this paper, we mostly look at results for the PATH Model. Due to the equivalence that we prove, those same results apply to the PRICE MODEL "for free".

In the PATH Model, the strategy of each node $v$ is still to choose a profit $\pi_{v}$. Define the $\pi$-length of a path $P$ to be $\sum_{v \in P} \pi_{v}$, and let $D_{\pi}(s, t)$ be the shortest $\pi$-length between nodes $s$ and $t$. Then, we can define a valid flow as follows.

Definition 3 (Valid flow for PATH MODEL). A valid flow $f$ given profits $\pi$ satisfies the following properties:

1. Flow only travels on shortest paths (in terms of $\pi$-length).

2. If $\lambda_{s t}>D_{\pi}(s, t)$, then s sends all of its $d_{s t}$ traffic to $t$. If $\lambda_{s t}<D_{\pi}(s, t)$, then it sends none of its traffic. (If $\lambda_{s t}=D_{\pi}(s, t)$ then any amount of traffic is allowed.) 
Note that in Definition 3 when $\lambda_{s t}=D_{\pi}(s, t)$, the source node $s$ can send any arbitrary amount of traffic and all the resulting flows will fall into the category of valid flow for the PATH MoDEL.

Proposition 1. A flow $f$ is a valid flow with respect to prices $p(\pi)$ in the PRICE Model exactly when it is a valid flow with respect to profits $\pi$ in the PATH MODEL.

Proof. By the construction of prices, $p_{w}^{t}$ is exactly the shortest $\pi$-distance from $w$ to $t$. Thus, saying that flow is forwarded to nodes with smallest $p_{w}^{t}$ is equivalent to saying that flow travels on shortest paths with respect to $\pi$-distance. Similarly, the shortest $\pi$-distance $D_{\pi}(s, t)$ from $s$ to $t$ is exactly equal to $\pi_{s}+p_{w}^{t}$ with $p_{w}^{t}$ being the smallest asking price of a neighbor of $s$. Thus, $\lambda_{s t}-p_{w}^{t}>\pi_{s}$ exactly when $\lambda_{s t}>D_{\pi}(s, t)$.

We define the utility of a source node for the PATH Model to be $U_{s}(\pi, f)=\pi_{s} \cdot f_{s t}$, where $f_{s t}$ is the amount of traffic $s$ sends in flow $f$. Similarly, the utility of a transit node $v$ is the amount of profit it obtains from forwarding traffic, i.e., $U_{v}(\pi, f)=\sum_{t}\left[\pi_{v} \cdot f_{t}^{i n}\right]$, where $f_{t}^{i n}$ is the amount of flow $v$ receives destined for destination $t$. If the total flow being transited by $v$ is more than its capacity $c_{v}$, then the utility of $v$ is defined to be negative infinity (from $v$ 's point of view, this means that $v$ should raise its desired profit $\pi_{v}$, and thus obtain less flow). We can now define a stable solution for the Path Model in exactly the same way as in the Price Model, the definition is identical to Definition 2, except with $p(\pi)$ replaced simply by $\pi$.

Definition 4 (Stable solution for PATH Model). A flow-profit pair $(f, \pi)$ is a stable solution if it satisfies the following properties:

1. $f$ is a valid flow with respect to $\pi$.

2. No node $v$ can change its desired profit $\pi_{v}$ and obtain a higher utility. Formally, let $\pi^{\prime}=$ $\left(\pi_{v}^{\prime}, \pi_{-v}\right)$ be the profit vector which is identical to $\pi$ except for node $v$ changing its desired profit to any $\pi_{v}^{\prime} \neq \pi_{v}$. Then, $U_{v}(\pi, f) \geq U_{v}\left(\pi^{\prime}, f^{\prime}\right)$, for every valid flow $f^{\prime} \in F\left(\pi^{\prime}\right)$.

We now show that the stable solutions in the PATH and the PRICE models have a strong relationship, and that if good stable solutions exist in the PATH model, then they are also stable in the PRICE model.

Proposition 2. If a flow-profit pair $(f, \pi)$ is a stable solution for the PATH MODEL, then it is also stable for the PRICE MODEL.

Proof. Consider a transit node $v$. First we will prove that if $v$ does not want to change its strategy (i.e., it is stable) under $(f, \pi)$ in one model, then it is also stable in the other model. Since the strategy space is the same, and due to Proposition 1, it is enough to show that the utility obtained by each node $v$ is exactly the same for any flow-profit pair $(f, \pi)$ in both the profit and pricing model, with $f$ being a valid flow with respect to $\pi$. This would mean that the set of possible deviations for node $v$ is the same in both models, and so if $v$ is stable in one, then it is stable in the other as well. The utility of node $v$ is $\sum_{t}\left[p_{v}^{t} \cdot f_{t}^{i n}-p_{w}^{t} \cdot f_{t}^{i n}\right]$ in the pricing model with $p_{w}^{t}$ being the cheapest outgoing price to $t$, which is exactly equal to $\sum_{t}\left[\pi_{v} \cdot f_{t}^{i n}\right]$ due to the way the prices are chosen. Thus, if a transit node is stable in one model, then it is also stable in the other.

We now show that if $(f, \pi)$ is a stable flow-profit pair in the profit model, then it is also stable for the pricing model. We already argued that all transit nodes will be stable, so consider a source node $s$. The utility of $s$ in the profit model is $U_{s}(\pi, f)=\pi_{s} \cdot f_{s t}$, where $f_{s t}$ is the amount of traffic 
$s$ sends in flow $f$. Suppose $f_{\text {st }}>0$. Since $f$ is a valid flow, this implies that $\lambda_{s t} \geq D$, where $D$ is the $\pi$-distance of the shortest path from $s$ to $t$. In fact, since $(f, \pi)$ is stable, then we know that $\lambda_{s t}=D$, since if $\lambda_{s t}$ were strictly smaller than $D$, then $s$ could increase $\pi_{s}$ by some amount and obtain larger utility. Due to the way prices are constructed in the pricing model, this means that $\lambda_{s t}=\pi_{s}+p_{w}^{t}$, where $p_{w}^{t}$ is the cheapest outgoing price to $t$ from $s$. Thus node $s$ is also stable in the pricing model: if it increases $\pi_{s}$ then its utility becomes 0 , since no flow will be sent because $s$ is asking for too much profit. If instead $s$ decreases $\pi_{s}$ and improves its utility (due to sending more flow), then it could have performed the same deviation in the profit model. To see this, consider that before lowering $\pi_{s}$, node $s$ has utility $\pi_{s} \cdot f_{s t}$. After lowering $\pi_{s}$ to $\pi_{s}-\epsilon$, node $s$ can obtain utility $\left(\lambda_{s t}-p_{w}^{t}\right) \cdot d_{s t}=\pi_{s} \cdot d_{s t}$ in the pricing model, or $\left(\pi_{s}-\epsilon\right) d_{s t}$ in the profit model. If $\pi_{s} \cdot d_{s t}>\pi_{s} \cdot f_{s t}$, then there must be some $\epsilon>0$ such that $\left(\pi_{s}-\epsilon\right) d_{s t}>\pi_{s} \cdot f_{s t}$. Thus, if lowering $\pi_{s}$ is an improving deviation for the pricing model, then it is also an improving deviation for the profit model, giving us a contradiction.

If on the other hand, $f_{s t}=0$, then due to $f$ being a valid flow it must be that $\lambda_{s t} \leq D$, where $D$ is as defined above. In fact, $\lambda_{s t} \leq D-\pi_{s}$, since otherwise $s$ could lower $\pi_{s}$ so that the shortest path becomes smaller than $\lambda_{s t}$, and thus obtain positive utility instead of zero. Due to the construction of prices, this means that $\lambda_{s t} \leq p_{w}^{t}$, and so the utility of node $s$ will be zero in both models no matter what choice of $\pi_{s}$ it makes.

This completes the proof of the fact that a stable $(f, \pi)$ is the profit model is also stable in the pricing model.

The converse of Proposition 2 is not true, but we can still show the following.

Proposition 3. If $(f, \pi)$ is a stable solution in the PRICE MODEL, then there exist profits $\pi^{\prime}$ such that $\left(f, \pi^{\prime}\right)$ is a stable solution in the PATH MODEL.

Proof. Suppose $(f, \pi)$ is stable in the pricing model. For each source node $s$, set the profits $\pi_{s}^{\prime}$ to be as follows. If $s$ is sending a positive amount of flow (i.e., $f_{s t}>0$ ), then set $\pi_{s}^{\prime}=\lambda_{s t}-p_{w}^{t}$, where $p_{w}^{t}$ is the amount node $s$ is paying to its neighbor $w$ to forward this flow. $\pi_{s}^{\prime}$ is nonnegative, and $\pi_{s}^{\prime} \geq \pi_{s}$, since otherwise this would not be a valid flow. For all other nodes, set $\pi_{v}^{\prime}=\pi_{v}$. We claim that $\left(f, \pi^{\prime}\right)$ is stable in the profit model.

First, notice that $f$ is a valid flow for $\pi^{\prime}$. No transit node would increase its $\pi_{v}^{\prime}$, since then it would get utility 0 due to the shortest path lengths being equal to $\lambda_{s t}$. It could decrease its $\pi_{v}^{\prime}$ and obtain more flow, but then it could have done the same in the pricing model with profits $\pi$. No source node $s$ with $f_{s t}>0$ would increase its $\pi_{v}^{\prime}$, since then it would get utility 0 . It could decrease its $\pi_{v}^{\prime}$ and obtain more flow, but then it could have done the same in the pricing model with profits $\pi$. Finally, a source node $s$ with $f_{s t}=0$ cannot gain anything from decreasing $\pi_{s}^{\prime}$, since otherwise it could have done that in the pricing model with profits $\pi$. This completes the proof.

From Propositions 2-3, the set of flows for which there exist stable prices is the same in both PATH Model and Price Model. Because of this, we will mostly focus on the PATH Model for the rest of the paper; and our results for the PATH MODEL will have immediate implications for the PRICE MODEL as well.

\section{Coalitional Stability of Networks of "Clouds"}

So far we defined only a simple model in which every player controls only a single node. This was done with the purpose of defining the basic concepts; our main goal, however, is to consider 
the more general case where a player may own an entire subnetwork of nodes (a "cloud"), or even several non-contiguous subnetworks. This is necessary to model ISPs as players, each of which may control one or more subnetworks ("domains" or Autonomous Systems (AS)). Also, a single player may control both transit and source nodes, and the utility of this player would consist of the total utility of the nodes under their control. Note that this also lets us handle cases where the same node (ISP) is both a source and a transit node (ISP), or is a source node for multiple destinations, etc. For any given set $S$ of nodes, we define the total utility of the nodes in the set $S$ as follows:

$$
U_{S}(f, \pi)=\sum_{v \in S} U_{v}(f, \pi)
$$

Now we define a coalitionally-stable solution:

Definition 5 (Coalitionally-stable solution). A flow-profit pair $(f, \pi)$ is coalitionally-stable if it satisfies the following properties:

1. $f$ is a valid flow with respect to $\pi$.

2. No set $S$ of nodes can change their desired profits $\pi$ and obtain a higher total utility. Formally, let $\pi^{\prime}=\left(\pi_{S}^{\prime}, \pi_{-S}\right)$ be the profit vector which is identical to $\pi$ except for nodes $v \in S$ changing their desired profit to any $\pi_{v}^{\prime}$. Then, $U_{S}(\pi, f) \geq U_{S}\left(\pi^{\prime}, f^{\prime}\right)$, for every valid flow $f^{\prime} \in F\left(\pi^{\prime}\right)$.

In other words, a coalitionally-stable solution is a solution such that no matter what subset of nodes falls under the control of a single entity (player), the current flow is still stable since this entity would not be able to increase its total utility by changing desired profits $\pi$. Note that this notion of a stable solution is very strong, strictly stronger than concepts such as Nash equilibrium. For example, there are many settings where no single node would want to change their price (this would be a Nash equilibrium), but a collection of nodes could collaborate and improve all their utilities by changing their prices simultaneously. A coalitionally-stable solution, however, is stable against such deviations as well. Moreover, it remains even if the ISP topologies (association of nodes/routers with ISPs) change, as long as the physical network topology remains unchanged. Despite this strong notion of coalitional stability, we show below that a stable solution always exists.

In our cloud model, the prices quoted (for any specific destination) by an ISP to different neighboring ISPs need not be the same, but will in general depend on each other. An ISP would choose profits at all of its internal nodes (routers), and the prices that it quotes on its interdomain connections will be composed from these chosen per-node profits, and the prices quoted by its neighboring ISPs, in a manner identical to that described in the previous section. This is illustrated in Figure 2.

We give the definitions of tight profits and tight flow-profit pair which we will use later to prove Proposition 4 and Lemma 1-2. Let us define tight profits in the PRICE MODEL to be profits such that for all sources $s, \pi_{s} \geq \lambda_{s t}-p_{w}^{t}$, where $p_{w}^{t}$ is the smallest price of any neighbor of $s$. A tight flow-profit pair $(f, \pi)$ is any flow-profit pair with tight profits.

Similarly to non-coalitional case, we have the following relationship between the coalitionallystable solutions in the PRICE and PATH models in the cloud settings.

Proposition 4. If a flow-profit pair $(f, \pi)$ is coalitionally-stable for the PATH MODEL, then it is also coalitionally-stable for the PRICE MODEL. 


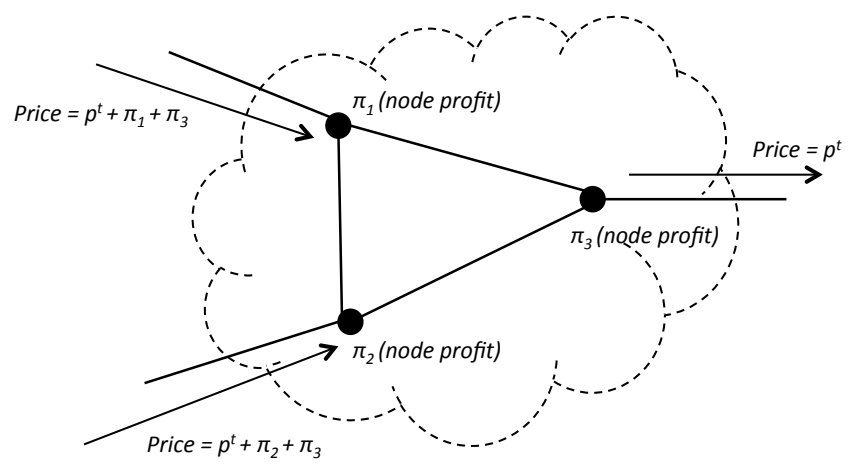

Figure 2: Example: profit choices and price dependencies in the cloud model. The numbers across the arrows represent the per-unit prices to be paid (in the direction of the arrows) towards sending traffic to destination $t$.

Before proving Proposition 4, we will first need to prove Lemma 1 and 2:

Lemma 1. In the PRICE MODEL, for any $(f, \pi)$ there is a tight $\left(f, \pi^{\prime}\right)$ such that $f$ is a valid flow for $\pi^{\prime}$ and for all nodes $v, U_{v}(f, \pi)=U_{v}\left(f, \pi^{\prime}\right)$.

Proof. For any source node $s$ with a profit $\pi_{s}$ which is not tight, simply set $\pi_{s}^{\prime}=\max \left\{\lambda_{s t}-p_{w}^{t}, 0\right\}$, so $\pi^{\prime}$ is clearly tight. First, we show that $f \in F\left(\pi^{\prime}\right)$. The prices at transit nodes do not change, so the flow is still forwarded to neighbors asking the cheapest price. Since $\pi^{\prime}$ is tight, then it never occurs that $\lambda_{s t}-p_{w}^{t}>\pi_{s}^{\prime}$. On the other hand, if $\lambda_{s t}-p_{w}^{t}<\pi_{s}^{\prime}$, then $\pi_{s}^{\prime}=0$ and $\lambda_{s t}<p_{w}^{t}$. Thus, it must be that $\lambda_{s t}-p_{w}^{t}<\pi_{s}$, since $\pi_{s} \geq 0$, and so $f$ does not send any flow from node $s$, as desired.

To confirm that all nodes obtain the same utility in both $(f, \pi)$ and $\left(f, \pi^{\prime}\right)$, note simply that utility for all transit nodes depends only on flow $f$ and prices, which are unchanged. For a source node $s$, the utility is $\lambda_{s t} f_{s t}-f_{s t} p_{w}^{t}$, which is unchanged.

Lemma 2. For a tight $(f, \pi)$, with $f$ a valid flow, the utility of every node $v$ is the same in both the PRICE and the PATH models.

Proof. For a transit node, the utilities are $U_{v}(\pi, f)=\sum_{t}\left[p_{v}^{t} \cdot f_{t}^{i n}-p_{w}^{t} \cdot f_{t}^{i n}\right]$ for the PrICE ModeL and $U_{v}(\pi, f)=\sum_{t}\left[\pi_{v} \cdot f_{t}^{i n}\right]$ for the PATH Model. Due to the construction of prices in PrICE Model, we have $p_{v}^{t}-p_{w}^{t}=\pi_{v}$, so these two versions of utilities are always equal.

For a source node, the utilities are $U_{s}(\pi, f)=\lambda_{s t} \cdot f_{s t}-p_{w}^{t} \cdot f_{s t}$ for the PRICE Model and $U_{s}(\pi, f)=\pi_{s} \cdot f_{s t}$ for the PATH Model. For $f_{s t}=0$, these are the same. If $f_{s t} \geq 0$, then since $f$ is a valid flow and $\pi$ is tight, it must be that $\pi_{s}=\lambda_{s t}-p_{w}^{t}$. Thus, the utility values are identical.

Proof of Proposition. We now proceed to proving the proposition 4 . Let $(f, \pi)$ be a coalitionallystable solution in the PATH Model. Since it is coalitionally-stable, we know that for every source $s$ with $\lambda_{s t}-\min _{w} D_{\pi}(w, t) \geq 0$ for $w$ being neighbors of $s$, we have that $\pi_{s}=\lambda_{s t}-\min _{w} D_{\pi}(w, t)$, since otherwise $s$ could increase $\pi_{s}$ and obtain more utility. Thus, when we consider $\pi$ in the PRICE MODEL, we see that $\pi$ is tight.

Consider an arbitrary deviation $\pi^{\prime}=\left(\pi_{S}^{\prime}, \pi_{-S}\right)$ by a set of nodes $S$, and suppose to the contrary that in the Price Model this is an improving deviation, i.e., $U_{S}\left(f^{\prime}, \pi^{\prime}\right)>U_{S}(f, \pi)$ for some $f^{\prime} \in F\left(\pi^{\prime}\right)$. Due to Lemma 1, there exists a tight profit vector $\pi^{\prime \prime}$ such that $f^{\prime} \in F\left(\pi^{\prime \prime}\right)$, and the utilities of all nodes are the same in both $\left(f^{\prime}, \pi^{\prime}\right)$ and $\left(f^{\prime}, \pi^{\prime \prime}\right)$. This profit vector $\pi^{\prime \prime}$ differs from 
$\pi^{\prime}$ only at nodes which are not tight in $\pi^{\prime}$. However, since $\pi$ is tight, the nodes not tight in $\pi^{\prime}$ must be nodes of $S$. Thus, we can think of $\pi^{\prime \prime}$ is a deviation of the set $S$ from the state $(f, \pi)$, i.e., $\pi^{\prime \prime}=\left(\pi_{S}^{\prime \prime}, \pi_{-S}\right)$. This deviation to $\left(f^{\prime}, \pi^{\prime \prime}\right)$ gives the same utilities to each node as in $\left(f^{\prime}, \pi^{\prime}\right)$, and so we still have that $U_{S}\left(f^{\prime}, \pi^{\prime \prime}\right)>U_{S}(f, \pi)$ in the PRICE Model. However, due to Lemma 2, and since both $\pi$ and $\pi^{\prime \prime}$ are tight, we know that the same holds in the PATH ModeL, which is a contradiction to $(f, \pi)$ being coalitionally-stable for the PATH ModeL.

From Proposition 4, if there exist good stable solutions in the PATH MODEL in cloud settings then there also exist good stable solutions for the PRICE MODEL in cloud settings. Thus in order to prove our main results Theorem 5.1 and Theorem 6.1, it is sufficient to focus on the PATH MODEL; as it will have immediate implications for the PRICE MODEL as well.

\section{Stability of Optimum Flows}

In this section we consider flows that maximize social welfare, and prove one of our main results that for both the PRICE and PATH models, there are profits which make these flows coalitionallystable. In other words, we prove that the price of stability is 1 even in the cloud setting: there are stable solutions which are as good as the social optimum, and so we do not need to sacrifice any social welfare in order to obtain stable prices, even in the cloud setting. First, let us investigate what the socially optimum solution looks like.

Define the social welfare of $(f, \pi)$ to be $\sum_{v} U_{v}(f, \pi)$. Since transfers of utility cancel out, in the Price Model this simply equals $\sum_{s t} \lambda_{s t} f_{s t}$, where $f_{s t}$ is the amount of flow sent from $s$ to $t$ in $f$. Thus, the socially optimal flow $f^{*}$ is simply the flow which obeys all node capacities $c_{v}$ and $d_{s t}$, and maximizes the total flow value $\sum_{s t} \lambda_{s t} f_{s t}$. It is easy to show that the socially optimum flow is the same in the PATH Model. Our main result of this section is the following theorem.

Theorem 5.1 (Existence of optimal stable solutions). For any flow $f^{*}$ maximizing social welfare, there exist profits $\pi$ such that $\left(f^{*}, \pi\right)$ is coalitionally-stable in both the PATH MODEL and the PRICE MODEL.

Proof. Consider a flow $f^{*}$ maximizing social welfare. Due to Proposition 4, it is sufficient to show that there exist profits such that $\left(f^{*}, \pi\right)$ is coalitionally-stable in the PATH ModeL. Thus in the rest of this proof, we will only deal with the PATH MODEL.

We give a brief outline of the proof. To begin with, we will state a linear program (LP) to compute the optimal flow and its dual LP. Then we will analyze the implications of complementary slackness conditions and show that the optimal flow is a valid flow if we set node profits equal to the values of dual variables. Finally, using the implications of complementary-slackness conditions, we will prove that setting node profits $\pi$ in such a way with $f^{*}$ being the flow implies that no coalition of nodes can change their profits to obtain a higher utility, thus proving that $\left(f^{*}, \pi\right)$ is coalitionally-stable.

Consider associating each path $p$ from a source node to a destination node with utility $\lambda_{p}$ (per unit flow sent on this path). This notion generalizes associating each source and destination pair $(s, t)$ with utility $\lambda_{s t}$. Now a path-based LP to compute optimal flow when we associate utilities 
with paths can be given by:

$$
\begin{aligned}
& \max _{p} \sum_{p} \lambda_{p} f_{p} \\
& \text { s.t. } \forall v: \quad \sum_{p \ni v} f_{p} \leq c_{v} \\
& \forall p: \quad f_{p} \geq 0
\end{aligned}
$$

This LP computes a flow $f^{*}$ corresponding to maximum social welfare such that the capacity constraints of each node are not violated (See Eqn 2). The dual LP corresponding to the primal LP (Eqn 1-3) is given by the following:

$$
\begin{aligned}
& \min _{v} \sum_{v} \pi_{v} c_{v} \\
& \text { s.t. } \forall p: \quad \sum_{v \in p} \pi_{v} \geq \lambda_{p} \\
& \forall v: \quad \pi_{v} \geq 0
\end{aligned}
$$

We claim that setting node profits exactly equal to their respective dual variables will stabilize $f^{*}$. We denote this set of node profits as $\pi^{*}$. To prove our claim, we first analyze the implications of complementary slackness conditions which $\left(f^{*}, \pi^{*}\right)$ satisfies. These conditions for the above primal-dual LP are given by:

$$
\begin{aligned}
& \forall v:\left(c_{v}-\sum_{p \ni v} f_{p}^{*}\right) \cdot \pi_{v}^{*}=0 \\
& \forall p: \quad\left(\sum_{v \in p} \pi_{v}^{*}-\lambda_{p}\right) \cdot f_{p}^{*}=0
\end{aligned}
$$

We make the following observations from the above complementary slackness conditions:

1. From Eqn 7, if a node $v$ has profit $\pi_{v}^{*}>0$ in $\left(f^{*}, \pi^{*}\right)$, then it must be saturated, i.e., the total flow carried by the paths of which $v$ is a part totals to exactly $c_{v}$.

2. From Eqn 7 , if a node $v$ is not saturated, then $\pi_{v}^{*}=0$ in $\left(f^{*}, \pi^{*}\right)$.

3. From Eqn 8, if a path $p$ carries a positive flow in $\left(f^{*}, \pi^{*}\right)$, then the $\pi$-length of path $p$ (i.e., the total profit of the nodes lying on this path) must be equal to the utility $\lambda_{p}$ corresponding to this path.

4. From Eqn 8, if $\pi$-length of path $p$ is strictly greater than $\lambda_{p}$ (note that it cannot be strictly less as per Eqn 5), then such a path carries no flow in $\left(f^{*}, \pi^{*}\right)$.

Now with the help of above observations, let us prove that $f^{*}$ is a valid flow for $\pi^{*}$.

Lemma 3. $f^{*}$ is a valid flow for $\pi^{*}$.

Proof. From Eqn 5, the $\pi$-length of any path in $\left(f^{*}, \pi^{*}\right)$ is at least $\lambda_{p}$. Combining this with observation 3 made above, the first condition of $f^{*}$ being a valid flow for $\pi^{*}$ is satisfied, i.e., flows in 
$\left(f^{*}, \pi^{*}\right)$ travel on shortest paths with respect to $\pi$-lengths. Now let us prove the second condition (see the definition of valid flows for the PATH Model). Let $D_{\pi}(s, t)$ be the $\pi$-length of a shortest path from a source node $s$ to a destination node $t$. From observation 4 above, if $D_{\pi}(s, t)>\lambda_{p}$ then $f_{p}=0$ and since $D_{\pi}(s, t)$ is never strictly less than $\lambda_{p}$ in $\left(f^{*}, \pi^{*}\right)$, the second and remaining condition for $f^{*}$ being a valid flow for $\pi^{*}$ is also satisfied.

Now that we have proved $f^{*}$ is a valid flow for $\pi^{*}$, the only part remaining to prove is that no set of nodes can change their profits to obtain a higher utility. First, let us notice that the total utility of nodes in a coalition $S$ can be expressed in two alternative forms by Equation 9 and 10:

$$
\begin{aligned}
U_{S}(f, \pi) & =\sum_{v \in S} U_{v}(f, \pi) \\
& =\sum_{v \in S} \pi_{v} \cdot \sum_{p \ni v} f_{p} \\
& =\sum_{p} f_{p} \sum_{v \in p: v \in S} \pi_{v}
\end{aligned}
$$

Eqn 9 follows from the definition of node utility in the PATH model and Eqn 10 follows from Eqn 9 by exchanging the order of summation. From Eqn 10, the total utility of nodes in a coalition can be interpreted as the sum of the profits the coalition earns from each path that passes through the coalition.

Now suppose to the contrary, that given $\left(f^{*}, \pi^{*}\right)$, there exists at least one coalition $S$ such that the nodes in $S$ can change their profits such that there exists at least one valid flow for the changed profits in which the total utility of nodes in the coalition $S$ increases. Denote by $\pi_{v}^{\prime}$ the changed profit of a node $v \in S$ in such a process (here $\pi_{v}^{\prime}=\pi_{v}^{*}$ for all $v \notin S$ ) and let $f^{\prime}$ denote a valid flow corresponding to these new node profits in which the nodes in the coalition obtain a strictly higher utility in total. Using this with Eqn 10, we get

$$
U_{S}\left(f^{\prime}, \pi^{\prime}\right)=\sum_{p} f_{p}^{\prime} \cdot \sum_{v \in p: v \in S} \pi_{v}^{\prime}>U_{S}\left(f^{*}, \pi^{*}\right)
$$

Let us denote $\sum_{v \in p: v \in S} \pi_{v}^{\prime}$ by $\pi_{p}^{\prime}$ and $\sum_{v \in p: v \in S} \pi_{v}^{*}$ by $\pi_{p}^{*}$. Thus we have

$$
U_{S}\left(f^{\prime}, \pi^{\prime}\right)=\sum_{p} f_{p}^{\prime} \cdot \pi_{p}^{\prime}
$$

Notice from the dual LP that $\pi$-length of any path in $\left(f^{*}, \pi^{*}\right)$ is at least $\lambda_{p}$. Hence if $\pi_{p}^{\prime}>\pi_{p}^{*}$ then the $\pi$-length of path $p$ exceeds $\lambda_{p}$, implying that $f_{p}^{\prime}$ must be zero as per the definition of valid flows. Hence Eqn 12 changes to:

$$
U_{S}\left(f^{\prime}, \pi^{\prime}\right)=\sum_{p: \pi_{p}^{\prime} \leq \pi_{p}^{*}} f_{p}^{\prime} \cdot \pi_{p}^{\prime} \leq \sum_{p} f_{p}^{\prime} \cdot \pi_{p}^{*}=\sum_{p}\left[f_{p}^{\prime} \sum_{v \in p: v \in S} \pi_{v}^{*}\right]
$$


Using similar reasoning as Eqn 9-10, we can express Eqn 13 as

$$
U_{S}\left(f^{\prime}, \pi^{\prime}\right) \leq \sum_{v \in S} \pi_{v}^{*} \cdot \sum_{p \ni v} f_{p}^{\prime}
$$

Now note that if the total utility of all the nodes in $S$ has to increase, then $\sum_{p \ni v} f_{p}^{\prime} \leq c_{v}$ for each $v \in S$, because if $\sum_{p \ni v} f_{p}^{\prime}>c_{v}$ then the utility of node $v$ becomes negative infinity. Also notice that whenever $\pi_{v}^{*}>0$, we have $\sum_{p \ni v} f_{p}^{*}=c_{v}$ from observation 1 made above from the complementary slackness conditions. Hence we have $\sum_{p \ni v} f_{p}^{\prime} \leq \sum_{p \ni v} f_{p}^{*}$ for any node $v \in S$ such that $\pi_{v}^{*}>0$. Using this in Eqn 14, we obtain

$$
\begin{aligned}
U_{S}\left(f^{\prime}, \pi^{\prime}\right) & \leq \sum_{v \in S} \pi_{v}^{*} \cdot \sum_{p \ni v} f_{p}^{*} \\
& =U_{S}\left(f^{*}, \pi^{*}\right)
\end{aligned}
$$

This contradicts our assumption that in $\left(f^{\prime}, \pi^{\prime}\right)$, the utility of the coalition $S$ strictly increases, thus proving our claim that $\left(f^{*}, \pi^{*}\right)$ is coalitionally-stable to complete the proof of Theorem 5.1.

Theorem 5.1 shows that the price of stability is 1: there are stable solutions which are as good as the social optimum, and so we do not need to sacrifice any social welfare in order to obtain stable prices, even in the cloud setting. In the next Section 6, we show that this results can be extended to the important case where instead of hard capacities $c_{v}$, the transit nodes have costs for transiting an amount of traffic $f_{v}$.

Remark 2: A careful look at the analysis in this section reveals that our results hold even if the utility that $s$ derives from sending traffic to $t$ is allowed to depend on the route this traffic takes. This is because the $\lambda_{p}$ values in our analysis depend on the paths, not just on source-destination pairs.

\section{Convex Transit Costs}

In this section we consider the case when instead of hard capacities $c_{v}$, the transit nodes have costs for transiting an amount of traffic $f_{v}$. These cost functions are assumed to be non-decreasing, convex, and continuously differentiable. Such cost functions often represent the internal motivations of ISP's much more accurately than hard capacities. Hard capacity constraints (as considered in the earlier sections) can also be incorporated into this framework by allowing the convex function approach infinity around the capacity limit $c_{v}$.

More formally, instead of capacities $c_{v}$, we are given cost functions $r_{v}$ for each node, with $r_{v}\left(f_{v}\right)$ being the cost faced by node $v$ for transiting $f_{v}$ amount of traffic. Everything else in this model is precisely the same as before. The only difference is that instead of transit node utility being $U_{v}(\pi, f)=\pi_{v} \cdot f_{v}$ when $f_{v} \leq c_{v}$ and $U_{v}(\pi, f)=-\infty$ otherwise, the transit node utility now is simply $U_{v}(\pi, f)=\pi_{v} \cdot f_{v}-r_{v}\left(f_{v}\right)$, where $f_{v}$ is the total amount of traffic being routed through $v$ in flow $f$.

All of our results hold for this version with convex transit costs instead of capacities. It is easy to see that Proposition 4 holds with an identical proof. Thus we focus on showing that Theorem 5.1 still holds for this case. To do this, first consider what the flow $f^{*}$ maximizing social welfare 
looks like. The sum of node utilities for the model with transit costs becomes

$$
\sum_{s t} \lambda_{s t} f_{s t}-\sum_{v} r_{v}\left(f_{v}\right)
$$

the payments between nodes cancel out as before. Let $f^{*}$ be the flow that maximizes the above quantity, maximizing the quality of the flow routed minus the total transit cost. The following theorem states our main result for this model.

Theorem 6.1 (Existence of optimal stable solutions for convex transit costs). For the model with convex transit costs instead of capacities, there exist profits $\pi$ such that $\left(f^{*}, \pi\right)$ is coalitionally-stable in both the PATH and the PRICE models. Here $f^{*}$ is a flow maximizing social welfare.

Proof. As it was the case with hard capacities (Theorem 5.1), it is enough to prove this for the PATH model. To form stable profits, we simply use marginal pricing as follows. For each transit node, set $\pi_{v}^{*}=r_{v}^{\prime}\left(f_{v}^{*}\right)$, where $f_{v}^{*}$ is the total amount of flow transited through $v$ in $f^{*}$, and $r_{v}^{\prime}$ is the derivative of $v$ 's cost function. For a source node $s$ with traffic destined to $t$, let $D$ be the $\pi^{*}$ distance from $s$ to $t$, not counting $\pi_{s}^{*}$. Then, set $\pi_{s}^{*}=\max \left(\lambda_{s t}-D, 0\right)$. In other words, we set $\pi_{s}^{*}$ so that $D_{\pi^{*}}(s, t) \geq \lambda_{s t}$.

Due to the fact that $f^{*}$ is socially optimal, we can now make the following observations. As in the proof of Theorem 5.1, $f_{p}^{*}$ is the flow sent on path $p$ in $f^{*}$, and $\lambda_{p}$ is shorthand for $\lambda_{s t}$ with $s$ and $t$ being endpoints of $p$.

1. Due to our choice of $\pi^{*}$, we know that the $\pi$-length of any path $p$ is at least $\lambda_{p}$.

2. If $f_{p}^{*}>0$, then $\sum_{v \in p} r_{v}^{\prime}\left(f_{v}^{*}\right) \leq \lambda_{p}$. If this were not the case, then we could decrease $f_{p}^{*}$ to obtain a solution better than the optimal one. Thus, due to our choice of $\pi^{*}$, we have that when $f_{p}^{*}>0$, then the $\pi$-length of path $p$ equals $\lambda_{p}$.

3. Also note the contrapositive of the above observation: if the $\pi$-length of path $p$ is strictly greater than $\lambda_{p}$, then $f_{p}^{*}=0$.

4. If a source node $s$ is not sending all of its flow (i.e., $\left.f_{s t}^{*}<d_{s t}\right)$, then $\sum_{v \in p} r_{v}^{\prime}\left(f_{v}^{*}\right) \geq \lambda_{p}$. Otherwise, we could increase $f_{p}^{*}$ to obtain a solution better than the optimal one. Thus, we have that if a source node $s$ is not sending all of its flow, then $\pi_{s}^{*}=0$.

Using the above observations, we can prove that $f^{*}$ is a valid flow for $\pi^{*}$ in a manner identical to Lemma 3. We must now show that no set of nodes $S$ can deviate from $\left(\pi^{*}, f^{*}\right)$ and obtain a total higher utility. Suppose to the contrary, that some coalition $S$ can change to $\pi^{\prime}$ and create a flow $f^{\prime}$ such that $U_{S}\left(\pi^{\prime}, f^{\prime}\right)>U_{S}\left(\pi^{*}, f^{*}\right)$. Most of the proof is identical to the proof of Theorem 5.1 , until we get to the equivalent of Inequality 14 :

$$
U_{S}\left(f^{\prime}, \pi^{\prime}\right) \leq \sum_{v \in S} \pi_{v}^{*} \cdot f_{v}^{\prime}-\sum_{v \in S} r_{v}\left(f_{v}^{\prime}\right)
$$

Here $f_{v}^{\prime}=\sum_{p \ni v} f_{p}^{\prime}$ is the total amount transited through node $v$ in flow $f^{\prime}$. Since $U_{S}\left(f^{\prime}, \pi^{\prime}\right)>$ $U_{S}\left(f^{*}, \pi^{*}\right)=\sum_{v \in S} \pi_{v}^{*} \cdot f_{v}^{*}-\sum_{v \in S} r_{v}\left(f_{v}^{*}\right)$, then it must be that for some node $v \in S$, it holds that

$$
\pi_{v}^{*} \cdot f_{v}^{*}-r_{v}\left(f_{v}^{*}\right)<\pi_{v}^{*} \cdot f_{v}^{\prime}-r_{v}\left(f_{v}^{\prime}\right) .
$$


We will show that Inequality 17 cannot hold, and thus obtain a contradiction. First consider a source $s$, with destination $t$. For source nodes, we assume that there is no cost for sending, so the above inequality simply becomes $\pi_{s}^{*} f_{s t}^{*}<\pi_{s}^{*} f_{s t}^{\prime}$. This can only occur when $f_{s t}^{*}<d_{s t}$, but due to Observation 4 above, this implies that $\pi_{s}^{*}=0$, and thus Inequality 17 cannot hold for source nodes.

Now consider a transit node $v$; we want to show that the function $g(x)=\pi_{v}^{*} \cdot x-r_{v}(x)$ is maximized at $x=f_{v}^{*}$, thus proving that Inequality 17 cannot hold. Note than $g$ is concave since $r_{v}$ is convex, and thus is maximized when $g^{\prime}(x)=0$. This occurs exactly when $x$ is such that $r_{v}^{\prime}(x)=\pi_{v}^{*}$. Since we set $\pi_{v}^{*}=r_{v}^{\prime}\left(f_{v}^{*}\right)$, this implies that $g(x)$ is maximized when $x=f_{v}^{*}$, as desired.

This contradicts our assumption that in $\left(f^{\prime}, \pi^{\prime}\right)$, the utility of the coalition $S$ strictly increases, thus proving our claim that $\left(f^{*}, \pi^{*}\right)$ is coalitionally-stable.

Remark 3: The coalitional equilibria from Theorem 5.1 and Theorem 6.1 fall under the category of Walrasian equilibria. As discussed in Sec 1.3 and Sec 2, these results are substantially stronger than Walrasian equilibria with similar marginal cost pricing schemes already investigated for many different models. This is because the coalitional equilibria in our model are also stable even when the nodes (and even coalitions) are allowed the freedom to change their prices instead of the typical assumption of exogenous prices. In fact, there are many settings where if a player is able to change the price of a good in a Walrasian equilibrium then it leads to loss of stability.

Remark 4: It is worth noting that while we have considered linear pricing so far, our stability results also hold for concave (and therefore sub-additive) pricing, where "volume discounts" are offered by the ISPs to their customers. It can be argued that an ISP can derive the same profit (that it can obtain through a concave pricing function) by using an appropriately defined linear pricing scheme. More precisely, this implies that when ISPs are allowed to choose any concave pricing scheme, there are price functions which make the optimum solution coalitionally stable. Moreover, these price functions are simple linear prices.

\section{Simulation Study}

The stability results in the previous sections implicitly assume that ISPs can correctly anticipate (or estimate) effect of their price changes on the resulting traffic patterns. Computing such an equilibrium exactly would in general require global network knowledge. In this section, therefore, we explore convergence of an incremental price update/discovery mechanism that may more closely represent price setting (updating) strategies used in practice. The impact of the price change (occurring at a relatively slow timescale) can be inferred by observing the changed behavior of the flows reacting to the price change, and therefore global network knowledge or sophisticated prediction algorithms may not be needed.

In the simulation study presented next, we consider two types of network topologies:

- Scale-free preferential attachment model-based topology: Here we use the BRITE (Boston university Representative Internet Topology gEnerator) simulator [56] to generate the network topology using the Barabasi-Albert model [6] of scale-free networks with preferential attachment. Preferential attachment based models are widely used to model Internet topology, e.g. see $[55,9,39,15]$. With BRITE-generated topologies, our main objective is to study the quality and convergence properties of stable solutions in the "cloud" settings - where each ISP is modeled as a subnetwork or a coalition of nodes, and the utility of an ISP is the combined utility of all the nodes it owns. 
- CAIDA topology: Here we use the open source database [58] from the Center of Applied Internet Data Analysis (CAIDA) for generating network topology. This database contains the connectivity information of $\approx 9000$ Autonomous Systems (AS's) in the internet. Previous work such as [53],[54], have studied the structural and temporal properties of the Internet using this dataset. In simulations using CAIDA dataset, our main objective is to study whether our observations on the efficiency of stable solutions extend to large realistic interdomain topologies, as well as study the profit distribution among the nodes (AS's) occupying different positions in the overall Internet. The internal topology of each AS is not contained in the CAIDA database. Moreover, to allow us to focus only on the effects of inter-domain topology (connectivity) and scale up to the large size of the inter-domain network, we here we model each AS as one with a fixed capacity for carrying the traffic (and within this capacity constraint it can route the traffic in any fashion between its neighbor ISPs), although we allow this capacity to vary.

In the following sections, we explain the simulation settings and results for each of the above cases.

\subsection{Scale-free preferential attachment topology}

In this section, we first describe topology generation, price-update strategies and more network specific settings before proceeding to presenting the results.

- Topology generation: When we model each ISP as a subnetwork, it translates to the overall network (representing the Internet) taking an hierarchical (two-level) structure consisting of: 1) The inter-ISP network topology, and 2) the intra-ISP network topology of routers, for each of the constituent ISPs. We use BRITE to generate both intra-ISP and inter-ISP network topologies based on the preferential attachment model. More specifically, we first generate intra-ISP subnetworks using BRITE topology generator and later in the second step again use the same topology generator to link these ISP networks. (In the second step, if the topology generator suggests an edge from ISP $i$ to ISP $j$ then in our simulation settings we put a link going from a randomly chosen node (router) owned by ISP $i$ to another randomly chosen node (router) owned by ISP $j$ ).

- Node strategies and dynamics: In our experiments, we choose a simple incremental profit update strategy for the nodes (See Section 3 for how it translates to prices on individual links). When considering a node $u$, we increment the profit $\pi_{u}$ by 1 unit, decrement $\pi_{u}$ by 1 unit, or keep it unchanged depending on which of these strategies maximizes the utility of the cloud that $u$ belongs to in the resulting flow. For simplicity, when there are multiple valid flows that can result with the new prices, our experiment chooses a new valid flow which is as similar as possible to the old flow. Note that each increment/decrement step, with accompanying utility calculations, involves computing shortest paths between all source-destination pairs for the new set of profits (prices).

We divide each experiment into rounds, where in each round, we consider all the nodes in a random sequence and update the profits as per the above incremental profit update policy. We stop the simulation when incrementing/decrementing profit of any of the nodes by 1 unit does not increase the utility of the ISP it belongs to. This notion of stability is with respect to the simple strategy of unit changes in the profit of the individual nodes, i.e., a coalition

of nodes may still benefit by changing (incrementing/decrementing) their profits by different amounts. 
- Network specifics: For each simulation instance, we generate a network with total number of transit nodes at 100, using the topology generation procedure described above, keeping the number of ISPs as 10 and each ISP owning 10 nodes from the network. We then choose 4 sink nodes at random and further add 28 source nodes to the network by forming a single link from each source node to a random transit node. We then assign these source nodes to each sink, with 7 sources to send traffic to each sink. We assume that each source is an independent subnetwork, representing customers of transiting ISPs.

The contribution $\lambda_{s t}$ to the social welfare made by a unit flow from a source $s$ to its assigned destination $t$ is set to a randomly chosen integer from $[1,10]$. We set the demand $d_{s t}=1$ of each source $s$ for its assigned destination $t$. We let the capacity $c_{v}$ of a sink node be 7 , i.e., the number of sources it can receive traffic from. We also set the capacity of every other transit and source node to 1 . This is done because for higher values of node capacities, the network is over-capacitated: most of the nodes (except edge nodes and well-connected nodes, see Section 7.2.1) may lose all the incentive to increase profits and all the flow gets routed through the network easily. Since Definition 1 leaves it unspecified what occurs when the outgoing price of a source node equals exactly $\lambda_{s t}$, we choose the tie-breaking rule for $s$ to send all of its $d_{s t}=1$ traffic in that case.

\subsubsection{Results}

We generated preferential attachment based network topologies as described above and performed simulation experiments to study efficiency and convergence properties of stable solutions. We describe the results below.

Efficiency of Solutions: We conducted simulations of our simple incremental profit update strategy for the above settings. We measured the social welfare (total network utility) of the converged solutions and compared them to that of the globally optimal utility (maximum social welfare). Furthermore, we studied the effect on the social welfare of varying inter-ISP connectedness keeping intra-ISP connectedness constant (with average intra-ISP degree $\approx 5$ ) and vice versa. We observed that on average, the social welfare was only 1.5 - 1.8 times away from the maximum social welfare. Thus, all the solutions to which the instances converged were of very good quality in terms of social wealfare (efficiency). Also, varying either intra-ISP or inter-ISP degree does not make a large difference to the quality of social welfare and it remains in the range shown in Table 1. As a further evidence, we simulated the case when each node is considered to be an ISP and varied the average degree of the nodes in the network. We observed that the social welfare still stays in a similar range, see Table 2. Thus the network density does not seem to play a significant role in the social efficiency of the converged solutions, and most of the converged solutions are close to the optimal network utility.

Convergence: All of our simulation instances in the above settings converged extremely quickly: convergence took less than $\approx 10$ rounds. Note that in each round, every node gets one chance to evaluate its strategy. See Figure 3 for histograms of the iterations it took the simulation instances to converge (for both cloud settings as well as the settings where we treat each node as an ISP). In addition, for single-node settings, where each node is an independent ISP, we considered the number of unstable nodes after each round. As Figure 4 shows (for a specific simulation instance), just after 3 or 4 rounds the majority of the nodes become stabilized, and convergence is attained in 6 rounds.

As seen from the above discussion, in our settings, the performance of networks with respect to 


\begin{tabular}{|c|c|c|c|}
\hline \hline Avg. inter-ISP Degree & Mean $\frac{S W_{\text {opt }}}{S W_{N E}}$ (std. dev.) & Avg. intra-ISP degree & Mean $\frac{S W_{N E}}{S W_{\text {opt }}}$ (std. dev.) \\
\hline 3 & $1.32(0.22)$ & 3 & $1.60 \quad(0.49)$ \\
4 & $1.59(0.58)$ & 4 & $1.62(0.7)$ \\
5 & $1.59(0.33)$ & 5 & $1.54(0.36)$ \\
6 & $1.58(0.33)$ & 6 & $1.60(0.39)$ \\
7 & $1.71(0.49)$ & 7 & $1.54(0.37)$ \\
8 & $1.68(0.39)$ & 8 & $1.64 \quad(0.59)$ \\
\hline
\end{tabular}

Table 1: ISPs modeled as clouds or subnetworks in preferential attachment networks: The above table shows the ratio of optimal social welfare (network utility) to social welfare attained upon convergence of the incremental price update strategy. The first column corresponds to the case of changing the average inter-ISP degree keeping the intra-ISP degree constant at 5 . The second column corresponds to the case of changing the average intra-ISP degree keeping the inter-ISP degree constant at 5 . Note that lower the ratio, closer is the social welfare (attained upon convergence) to the optimal.

\begin{tabular}{|c|cc|}
\hline \hline Avg. node degree & Mean $\frac{S W_{\text {opt }}}{S W_{N E}}$ (std. dev.) \\
\hline 4 & 1.82 & $(0.40)$ \\
8 & 1.76 & $(0.39)$ \\
10 & 1.83 & $(0.48)$ \\
15 & 1.67 & $(0.39)$ \\
25 & 1.39 & $(0.22)$ \\
30 & 1.41 & $(0.17)$ \\
\hline
\end{tabular}

Table 2: The table shows the variation in the average ratio of the optimal social welfare (network utility) to the social welfare (network utility) attained on convergence, for preferential attachment networks with each single node modeling an independent ISP. The total number of nodes (ISPs) in the network is set to 100. Note that despite a large variation in the average node degree, the social efficient attained upon convergence remains relatively consistent. Note that lower the ratio, closer is the social welfare to the optimal.
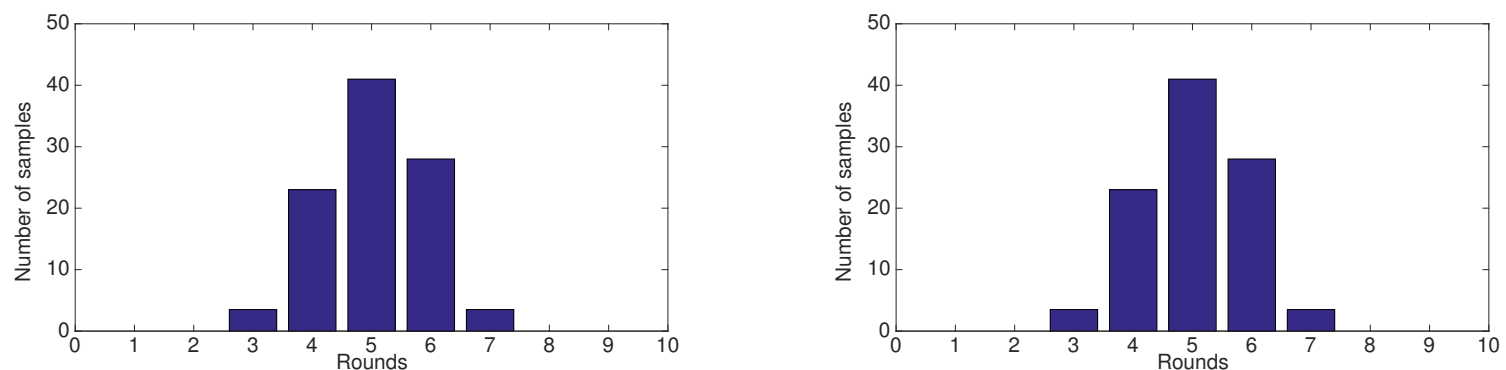

Figure 3: Left side: Histogram of the rounds taken for the experiments to converge with scale-free preferential attachment connectivity in cloud settings. Two sets of experiments have been aggregated in this histogram: one set corresponds to keeping inter-ISP degree constant at 5 and varying mean intra-ISP degree between 3 and 8 , and another set corresponds to keeping intra-ISP degree constant at 5 and varying mean inter-ISP degree between 3 and 9 . Right side: Histogram of rounds taken for the experiments to converge with with scale-free preferential attachment connectivity with average degree 9 , when each ISP is modeled as a single node with a capacity constraint. 


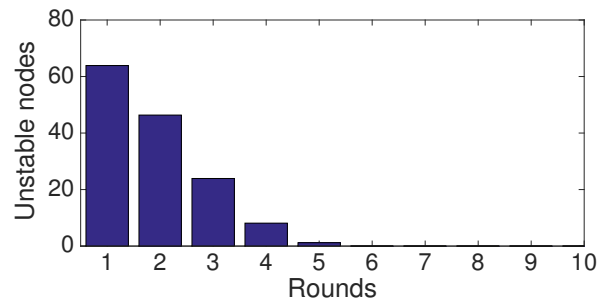

Figure 4: The number of unstable nodes after each round decreases quickly, with scale-free preferential attachment networks. The figure corresponds to the statistic when the average degree is 10; for all other degrees a similar pattern was observed.

convergence and the quality of stable solutions remains nearly same whether we consider ISPs as subnetworks or ISPs as single nodes. Thus for the CAIDA-topology based network that we consider in the next subsection, we restrict ourselves to the inter-domain topology only, i.e., each ISP or AS is represented as a single node (with an individual capacity constraint) in the overall inter-domain network.

\subsection{Inter-domain topology from the CAIDA database}

First we first describe the topology, node strategies (and dynamics) and more network specific settings before proceeding to presenting the results.

- Topology: The online database courtesy CAIDA contains Autonomous System-level (or ASlevel) topology information of about 9000 AS's. In our set of experiments with this topology (which we loosely refer to as the 'CAIDA topology'), we create a graph of nodes representing the CAIDA topology, with each node representing an independent AS. Here each AS is assumed to be a subnetwork or cloud into itself, but with the flexibility of routing the traffic between neighboring AS's in any fashion (subject to a constraint on the total traffic it carries, as described below). For each connection between AS $u$ and $v$ in the database, we add two directional edges between the corresponding nodes (one edge from $u$ to $v$ and another from $v$ to $u$ ) in the graph (the database does not specify the direction of traffic exchange between the AS's).

- Node strategies and dynamics: We use a similar incremental profit update strategy as we used for the preferential attachment case (See Sec 7.1). Similar to the settings in preferential attachment based networks, while considering a node $u$, we increment the profit $\pi_{u}$ by 1 unit, decrement $\pi_{u}$ by 1 unit, or keep it unchanged - however this decision depends only on the utility of the individual node under consideration instead of a subnetwork (since the intraAS topology is abstracted away, representing the AS as a single node with a total capacity constraint). We divide the experiments into rounds similar to the preferential attachment case described in Section 7.1, and consider an instance converged when no node (AS) benefits by incrementing/decrementing its profit.

- Network specifics: After generating the network as described above, we then choose 9 sink nodes at random from the network and further add 45 source nodes to the network by forming a single link from each source node to a random transit node. We then assign these source nodes to each sink, with 5 sources to send traffic to each sink. We assume that each source is 


\begin{tabular}{|c|cc|}
\hline \hline Node capacity & Mean $\frac{\sum \lambda_{s t} d_{s t}}{S W_{N E}}$ (std. dev) \\
\hline 1 & 2.97 & $(0.74)$ \\
2 & 1.55 & $(0.17)$ \\
3 & 1.22 & $(0.03)$ \\
4 & 1.1 & $(0.04)$ \\
5 & 1.03 & $(0.02)$ \\
\hline \hline
\end{tabular}

Table 3: The above table shows how close the social welfare of the instances is to $\sum \lambda_{s t} d_{s t}$ (which upper bounds the optimal social welfare) for the networks based on CAIDA topology with each node in the topology representing an independent AS.

an independent subnetwork or AS into itself. The source utility variables $\lambda_{s t}$ and the source demands are set in the same way as in the preferential attachment case. As to the capacities on the total traffic an AS can carry, we use various settings as described in the results section to take the network from the under-capacitated to the over-capacitated region.

Note that while 50 source-destination pairs is very small considering the Internet context, we had to choose a small number to keep the complexity reasonable. To evaluate increment/decrement strategy of a node by means of computing shortest paths for all sourcedestination pairs it takes $O\left(n_{s t} \cdot|E|\right)$ computation time, with $n_{s t}$ being the number of source-destination pairs, and $|E|$ the number of edges in the graph. Thus each round takes $O\left(n \cdot n_{s t} \cdot|E|\right)$ computation time (where $n$ is the number of nodes in the network), which can be very high for networks as large as the CAIDA topology. We have however conducted limited number of simulations for up to 200 source-destination pairs, and observed the results described in the later part of this section hold. Since the number of source-destination pairs is small relative to the actual Internet, we believe that the convergence time (round) results that we obtained in this case are not truly representative quantities. In this section, therefore, we focus only on studying the efficiency of the converged solutions, as well as profit sharing between ISPs (AS's) with different connectivity levels.

\subsubsection{Results}

Efficiency of Solutions: Now we present the results about the social welfare (total network utility) of the converged solutions. We found that computing global optimal utility for an instance in this case to be computationally challenging (with our graph containing $\approx 9 k$ nodes, $\approx 60 k$ edges). Hence instead of comparing the maximum social welfare to the globally optimal utility, we compare it with the upper bound on the total utility that can be obtained in the simulation instances (and this upper bound is simply $\sum_{(s t)} \lambda_{s t} d_{s t}$, where $\lambda_{s t}$ are the source utilities and $d_{s t}$ are the source demands).

We varied the node degrees and compared the social welfare of the converged solutions with the above upper bound on total utility. Table 3 shows the results of this comparison. We see that as the network goes from the under-capacitated state (where each node has small capacity to carry the traffic) to the over-capacitated state, the quality of social welfare approaches the upper bound on the total utility. For example, when all node capacities are set to 1, this ratio is 2.97 which decreases to nearly 1.03 when all node capacities are 5 . While this is not surprising for the overcapacitated case, even for under-capacitated case it is within reasonable proximity to the globally optimal social welfare. Recall that this ratio is computed with respect to an upper bound of the 
optimum, so the true ratio (computed with respect to the optimum) would be smaller, implying a closer approximation. We also performed experiments with randomized node capacities (e.g., randomly chosen integer values between 1-3 or 1-5 units) but it was observed to not have a significant effect on the social welfare compared to setting the capacities constant.

Profit distribution: In our simulations, we also calculated the distribution of profits with respect to their degree, which is a measure of how influential a position a node occupies in the network. We present the results by dividing the nodes in 4 categories: leaf nodes (which includes sources) having degree 1 , nodes with small degree with degree $\in[2,10]$, nodes with medium degree (degree $\in[11,100]$ ), nodes with large degree (degree $\in[101,1000])$ and nodes with extremely large degree (degree more than 1000).

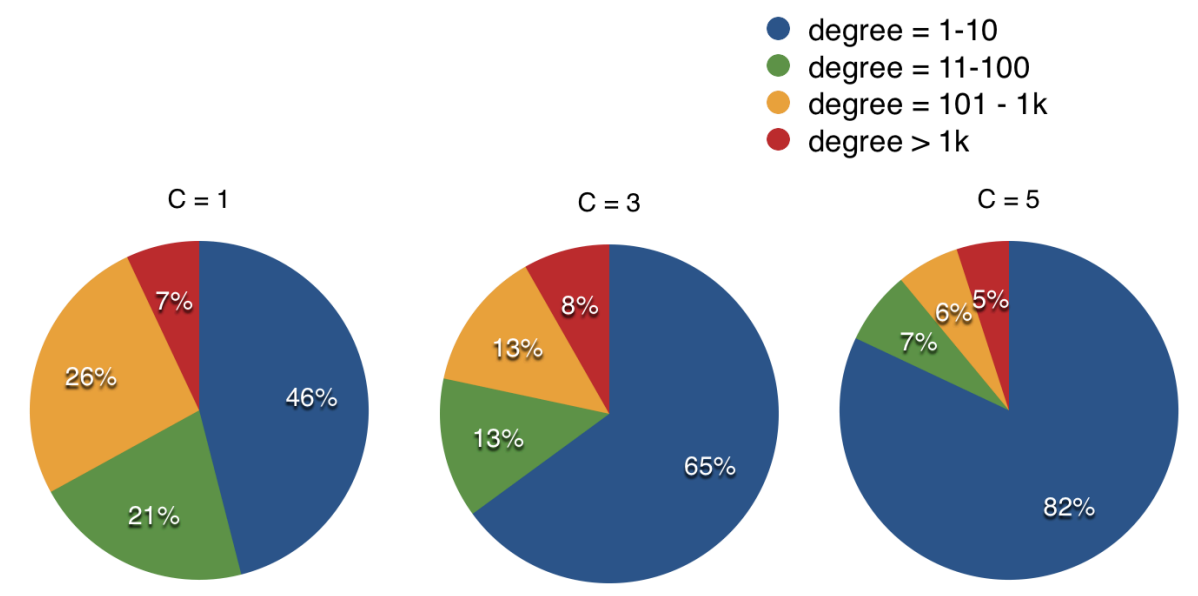

Figure 5: The profit distribution among the nodes (AS's) of various categories: The leftmost pie-chart corresponds to the under-capacitated case of all node capacities set to 1 . In the middle pie-chart, all node capacities are set to 3 whereas the rightmost pie-chart corresponds to over-capacitated case with all node capacities set to 5 . As seen from the plot, going from the under-capacitated to the over-capacitated network state increases the profit share of nodes nodes lying on the edge of the network (degree $1-10$ ) but shrinks the share of the "middlemen" nodes (degree $11-1000)$, whereas the profit share of very-highly connected nodes remain relatively consistent.

The plots in Figure 5 show the share of the total profit obtained by each category of nodes. We see that as we go from the under-capacitated to the over-capacitated networks, the share of the profit obtained by small degree nodes increases while the medium-to-large degree nodes see their profits shrinking (very large degree nodes maintain a reasonably consistent share in all cases). This is perhaps because the medium-to-large degree nodes are the nodes which face competition for multiple high-capacity routes when the network starts going from the under-capacitated to the over-capacitated state. Leaf and small-degree nodes are most likely close to the edge of the network, thus they face only a little competition. Extremely-large degree nodes occupy central influential positions, facing little or no competition (as most of the paths go through at least one of them, they can afford to increase their profits and yet maintain their share of total profit). Thus we find that it is the "weak middlemen" nodes who face the pressure for keeping their profits low in the presence of multiple high-capacity routes in the setting of having an over-capacitated network. In addition, as seen from Table 4 although the nodes not central to the network (degree 1-10) obtain 
a large share of the profit as the network becomes over-capacitated, individually their profit share is low due to them being very large in number and not occupying central position in the network. However, the nodes with high or very-high connectivity (degree 1000 and more) continue to earn high individual profit in both under-capacitated as well as over-capacitated settings due to their influential position in the network, i.e., most of the paths must pass through these nodes.

\begin{tabular}{|c|c|c|c|c|}
\hline \hline \multirow{2}{*}{ Node degree } & \multirow{2}{*}{ Number of nodes } & \multicolumn{3}{|c|}{ \%profit per node } \\
\cline { 3 - 5 } & & $c=1$ & $c=3$ & $c=5$ \\
\hline $1-10$ & 8542 & 0.02 & 0.025 & 0.033 \\
$11-100$ & 650 & 0.03 & 0.02 & 0.01 \\
$101-1000$ & 53 & 0.49 & 0.26 & 0.12 \\
$>1000$ & 4 & 1.75 & 2.21 & 1 \\
\hline \hline
\end{tabular}

Table 4: The above table shows the percentage of profits earned by individual node (AS) depending on their degree in the network based on CAIDA topology. Note that although nodes on the network edge (degree 1-10) earn majority of the profits combined together (Fig. 5), their individual share is miniscule because of their large number. However, the nodes central to the network (with high to very-high degree) earn a significant profit share individually (as expected) because of a large number of routes passing through them.

\subsubsection{Concave Pricing}

For CAIDA topology, we also carried out simulations with concave piecewise-linear pricing, where a node gives a "volume discount" to its customer nodes, i.e., the price per unit traffic charged by a node to any of its customer nodes decreases as the amount of traffic sent to it by the customer node increases. For the simulations in this case, topology and network specifics remain the same as described in Section 7.2 except that the source capacities (i.e., the maximum amount any given source can send) are now randomly chosen integers from $[1,10]$.

The node pricing strategies that we use in our study are motivated by the concave piecewiselinear transit pricing scheme described in Table I of [59]. For our simulations, the traffic breakpoints belong to the set $\{0,2,4,8\}$, i.e., a node decides its target profit per unit traffic sent to it by a customer node depending on which interval the amount of traffic sent falls into, with the intervals being $[0,2),[2,4),[4,8),[8,10]$. This suffices because the source capacities are random integers chosen from $[1,10]$ for our experiments. For a node, say $u$, if $t$ denotes the amount of traffic sent by a customer node through $u$ and if $y$ denotes the target profit of $u$ for the traffic interval $[0,2)$ then a node chooses its profit $\pi_{u}(t)$ as described below:

$$
\pi_{u}(t)= \begin{cases}y & \text { if } t \in[0,2) \\ 3 y / 5 & \text { if } t \in[2,4) \\ 2 y / 5 & \text { if } t \in[4,8) \\ y / 5 & \text { if } t \in[8,10]\end{cases}
$$

The incremental update strategy for nodes is similar to that in Section 7.2, except now a node $u$ can increment or decrement its target profit for the base traffic interval $[0,2)$ by 1 unit (and updating $\pi_{u}(\cdot)$ accordingly) or can keep $\pi_{u}(\cdot)$ unchanged depending on which of these strategies maximize its utility.

We divide each experiment into rounds as before. We found that the efficiency of the solutions as well as the profit distribution to which the instances converge were similar to that in the linear 


\begin{tabular}{|c|cc|}
\hline \hline Node capacity & Mean $\frac{\sum \lambda_{s t} d_{s t}}{S W_{N E}} \quad$ (std. dev) \\
\hline 10 & 2.27 & $(0.19)$ \\
20 & 1.35 & $(0.11)$ \\
30 & 1.13 & $(0.05)$ \\
50 & 1.06 & $(0.04)$ \\
\hline \hline
\end{tabular}

Table 5: The above table shows how close the social welfare of the instances is to $\sum \lambda_{s t} d_{s t}$ (which upper bounds the optimal social welfare) with concave non-linear pricing for the networks based on CAIDA topology with each node in the topology representing an independent AS.

pricing case in Section 7.2.1. Table 5 shows the comparison between the social welfare of the instances with the upper bound on the total utility that can be obtained in the simulation instances (and this upper bound is simply $\sum_{(s t)} \lambda_{s t} d_{s t}$, where $\lambda_{s t}$ are the source utilities and $d_{s t}$ are the source demands). The results are shown in Table 5 where we observe that as the network goes from the under-capacitated state (i.e., when all transit node capacities are set to 10) to the over-capacitated state (i.e., when all transit node capacities are set to 50), the quality of the social welfare approaches the upper bound on the total utility. Profit distribution results were also observed to be similar to the results in Section 7.2.1 and we omit them for the sake of brevity.

\section{Concluding Remarks and Future Directions}

In this paper, we have modeled and analyzed stable and efficient pricing for inter-domain traffic routing in the Internet, under the assumptions that ISPs are solely interested in maximizing their own profit, and can estimate the effect of the prices they quote on the resulting traffic patterns. Using no assumptions about the network topology, we prove that there exists a pricing scheme that attains network-wide efficiency and is yet coalitionally stable, where the coalitions correspond to the ISPs that are acting in self-interest. In other words, the pricing scheme not only maximizes the overall utility of the resulting traffic flows, but is also such that ISPs cannot expect to improve their profit through deviation from it. This is quite significant, since we consider a much stronger notion of coalitional stability than related literature, along with allowing multiple destinations. Since our complete information game model implicitly assumes that ISPs can correctly anticipate the effect of their price change on the traffic, we evaluate through simulations an incremental best-response based simple price update policy that can be implemented without knowledge of the network, and is motivated by incremental price update/discovery mechanisms used in a typical marketplace. We observe that this incremental price update policy always converges, and yield a solution that is near-optimal in terms of network utility in most cases. Analytically bounding the worst-case behavior of coalitionally stable solutions remains an open problem, however.

As mentioned in Section 1, a restriction of our model is the directionality of the payments, or that only one endpoint should receive utility from the communication. This is the primary reason why the model does not correctly reflect the current Internet practices. While we have discussed earlier how our model could apply to some of the proposed inter-domain routing architectures for the future Internet, or emerging traffic patterns (in the current Internet) between cloud computing servers and client, the extension of our framework to more general Internet practices and applications remains an open question.

The pricing framework that we consider falls within the framework of point-to-destination pricing, which has been envisioned in some recent work such as [52]. If the ratio of the traffic that a 
source sends to different destinations remains invariant as price changes, then we believe that our results can be extended to point-to-anywhere pricing. A full exploration of this issue will however be pursued in future work. Also note that we have assumed linear source utilities in this model; while our stability results extend to concave price functions, the extension to non-linear (concave) source utilities is not direct and requires further investigation.

Finally, note that the convergence of our price update policy have been evaluated in a moreor-less uniform updating scenario, where our incremental best-response based price updates are implemented in rounds. A large disparity in its implementation across different players (ISPs), in terms of frequency or size of updates, can potentially affect the convergence properties of the policy in significant ways. A thorough investigation of this issue also remains open for future study.

\section{Acknowledgments}

This work was supported by the National Science Foundation through award CNS-1218374.

\section{References}

[1] E. Anshelevich, A. Hate, and K. Kar. "Strategic Pricing in Next-hop Routing with Elastic Demands." In Proc. 4th International Symposium on Algorithmic Game Theory (SAGT 2011).

[2] D. Acemoglu, R. Johari, and A.E. Ozdaglar, "Partially optimal routing," IEEE Journal on Selected Areas in Communications 25 (6): 1148-1160 (2007).

[3] D. Acemoglu and A. Ozdaglar, "Competition in Parallel-Serial Networks," IEEE Journal on Selected Areas in Communications, 25(6):1180-1192 (2007).

[4] E. Anshelevich and G. Wilfong, "Network Formation and Routing by Strategic Agents using Local Contracts," Proc. 4th International Workshop On Internet And Network Economics (WINE 2008).

[5] S. Balon and G. Leduc, "Combined intra- and inter-domain traffic engineering using hot-potato aware link weights optimization," Proc. SIGMETRICS '08, pages 441-442, 2008.

[6] A-L. Barabasi and R. Albert, "Emergence of scaling in random networks," Science 286 (5439): 509-512 (October 1999).

[7] T. Basar, and R. Srikant, "A Stackelberg network game with a large number of followers," Journal of Optimization Theory and Applications, 115(3): 479-490, (2002).

[8] T. Basar and R. Srikant, "Revenue-maximizing pricing and capacity expansion in a many-users regime," Proc. of IEEE INFOCOM, 2002.

[9] T. Bu, Tian, and D. Towsley, "On distinguishing between Internet power law topology generators," Proc. IEEE INFOCOM 2002.

[10] I. Castro, A. Panda, B. Raghavan, S. Shenker, and S. Gorinsky, "Route Bazaar: Automatic Interdomain Contract Negotiation", Proc. USENIX HotOS XV, May 2015.

[11] S. Chawla and F. Niu. The Price of Anarchy in Bertrand Games. Proc. 10th ACM Conference on Electronic Commerce (EC 2009). 
[12] S. Chawla and T. Roughgarden, "Bertrand Competition in Networks," Proc. 1st Intl. Symp. on Algorithmic Game Theory (SAGT '08), 2008.

[13] R. Cole and Y. Dodis and T. Roughgarden, "Pricing network edges for heterogeneous selfish users," Proc. of STOC, 2003.

[14] J.R. Correa, R. Lederman, N.E. Stier-Moses, "Pricing with markups under horizontal and vertical competition," Proceedings of the Behavioral and Quantitative Game Theory: Conference on Future Directions, New York (2010).

[15] A. Dhamdhere, and C. Dovrolis. "Twelve years in the evolution of the internet ecosystem," IEEE/ACM Transactions on Networking (ToN) 19, no. 5 (2011): 1420-1433.

[16] N. Feamster, J. Borkenhagen, and J. Rexford, "Guidelines for interdomain traffic engineering," SIGCOMM Comput. Commun. Rev., vol. 33, no. 5, pp. 19-30 (2003).

[17] L. Fleischer, K. Jain and M. Mahdian, "Tolls for heterogeneous selfish users in multicommodity networks and generalized congestion games," Proc. of FOCS, pp. 277-285, 2004.

[18] L. Gao, "On Inferring Autonomous System Relationships in the Internet", IEEE/ACM Transactions on Networking (ToN), 9(6), pp. 733-745, 2001.

[19] R. Gao, C. Dovrolis and E. W. Zegura, "Interdomain Ingress Traffic Engineering Through Optimized AS-Path Prepending," NETWORKING 2005: 647-658.

[20] P. Godfrey, I. Ganichev, S. Shenker, and I. Stoica, "Pathlet Routing," Proc. SIGCOMM, 2009.

[21] A. Hall, E. Nikolova, and C. Papadimitriou, "Incentive-Compatible Interdomain Routing with Linear Utilities," Internet Mathematics. 5(4): 395-410 (2008).

[22] A. Hayrapetyan, É. Tardos, and T. Wexler. A network pricing game for selfish traffic. In Distributed Computing, March 2007.

[23] L. He, J. Walrand, "Pricing Internet Services With Multiple Providers", Proc. 41st Annual Allerton Conf. on Communication, Control, and Computing, Monticello, IL, 2003.

[24] J. He et al., "Rethinking internet traffic management: from multiple decompositions to a practical protocol," Proc. CoNEXT 'O\%.

[25] X. Huang, A. Ozdaglar and D. Acemoglu, "Efficiency and Braess Paradox under pricing in general networks," IEEE Journal on Selected Areas in Communication, vol. 24, no. 5, pp. 977-991, 2006.

[26] G. Huston, "Interconnection, peering, and settlements," Proc. Internet Global Summit, The Internet Society, San Jose, CA, June 1999.

[27] N. Immorlica, D. Karger, E. Nikolova, and R. Sami. "First-Price Path Auctions." Proc. EC 2005.

[28] R. Johari and J.N. Tsitsiklis. Routing and peering in a competitive Internet. IEEE Conference on Decision and Control (CDC), 2004. 
[29] F. Kelly, A. Maulloo and D. Tan, "Rate control for communication networks: shadow prices, proportional fairness and stability", Journal of the Operational Research society, pp. 237-252 (1998).

[30] V. Kuleshov and G. Wilfong, "Bertrand and Cournot Competition in Two-Sided Markets," Unpublished manuscript, www.cs.mcgill.ca/ vkules/cournot.pdf.

[31] C. Labovitz et. al., "Internet Inter-Domain Traffic," Proc. of ACM SIGCOMM, New Delhi, August 2010.

[32] H. Levin, M. Schapira and A. Zohar, "Interdomain Routing and Games," SIAM J. Comput. 40(6):1892-1912 (2011).

[33] P. Maille and B. Tuffin, "Analysis of Price Competition in a Slotted Resource Allocation Game," Proceedings of IEEE INFOCOM 2008, April 2008.

[34] E. Markakis and A. Saberi, "On the Core of the Multicommidity Flow Game", Decision Support Systems, 39(1): 3-10 (2005).

[35] A. Mas-Colell, M. Whinston, and J. Green. Microeconomic Theory. Oxford, 1995.

[36] A. Ozdaglar, "Price competition with elastic traffic," Networks, 52(3):141-155 (2008).

[37] A. Ozdaglar and R. Srikant, "Incentives and Pricing in Communication Networks," Chapter 22 in: N. Nisan et. al., Algorithmic Game Theory, Cambridge University Press.

[38] C. Papadimitriou and G. Valiant, "A New Look at Selfish Routing," Innov. in Computer Science, 2010.

[39] S. Park, D. Pennock, and C. Giles, "Comparing static and dynamic measurements and models of the Internet's AS topology," Proc. IEEE INFOCOM 2004.

[40] B. Quoitin et al., "Interdomain Traffic Engineering with BGP," IEEE Comm. Magazine, vol. 41 (2003).

[41] B. Quoitin, S. Tandel, S. Uhlig and O. Bonaventure, "Interdomain traffic engineering with redistribution communities," Computer Communications 27, pp. 355-363 (2004).

[42] Y. Rekhter and T. Li, "A Border Gateway Protocol 4 (BGP-4)", RFC 4271, January 2006.

[43] T. Roughgarden and É. Tardos, "How Bad is Selfish Routing?," Journal of the ACM, 49(2): 236-259 (2002).

[44] M. Schapira, Y. Zhu, and J. Rexford, "Putting BGP on the right path: A case for next-hop routing," Proc. HotNets-IX, Monterey, CA, October 2010.

[45] J. L. Sobrinho, "Network routing with path vector protocols: Theory and applications," Proceedings of ACM SIGCOMM '03, Karlsruhe, Germany, Aug. 2003.

[46] J. Sommers, P. Barford, N. Duffield, and A. Ron, "Accurate and Efficient SLA Compliance Monitoring," In Proceedings of the ACM Special Interest Group on Data Communication (SIGCOMM), 2007. 
[47] G. Srimali, A. Akella, A. Mutapcic, "Cooperative Interdomain Traffic Engineering Using Nash Bargaining and Decomposition," IEEE/ACM Transactions on Networking, 18(2): 341-352 (2010).

[48] V. Valancius, C. Lumezanu, N. Feamster, R. Johari, and V. V. Vazirani, "How many tiers?: pricing in the internet transit market," Proceedings of the ACM SIGCOMM 2011, pp. 194-205.

[49] Y. Xi and E. M. Yeh, "Pricing, competition, and routing in relay networks," Proc. 47th Annual Allerton conference on Communication, Control, and Computing, 2009.

[50] W. Xu and J. Rexford, "MIRO: multi-path interdomain routing," SIGCOMM Computer Communications Review, Vol. 36, No. 4 (2006).

[51] Y.R. Yang et al., "On route selection for interdomain traffic engineering," IEEE Network, 19(6): 20-27 (2005).

[52] M. Yuksel, A. Gupta, K. Kar, and S. Kalyanaraman, "Contract-Switching for Managing InterDomain Dynamics," In Next-Generation Internet Architectures and Protocols, Eds. B. Ramamurthy et. al., Cambridge University Press, pp.136-153, December 2010.

[53] P. Mahadevan, D. Krioukov, M. Fomenkov, X. Dimitropoulos, and A. Vahdat, "The Internet AS-level topology: three data sources and one definitive metric," ACM SIGCOMM Computer Communication Review 36, no. 1 (2006): 17-26.

[54] R. Oliveira, B. Zhang, and L. Zhang, "Observing the evolution of Internet AS topology," In ACM SIGCOMM Computer Communication Review, vol. 37, no. 4, pp. 313-324. ACM, 2007.

[55] A. Vazquez, R. Pastor-Satorras, and A. Vespignani, "Large-scale topological and dynamical properties of the Internet," Physical Review E 65, no. 6 (2002): 066130.

[56] The BRITE simulator, http://www.cs.bu.edu/brite/

[57] http://bgp.potaroo.net/cidr/autnums.html

[58] The Skitter AS Links Dataset, http://www.caida.org/data/active/skitter_aslinks_dataset.xml, (Accessed January 27, 2015)

[59] I. Castro, R. Stanojevic, and S. Gorinsky, "Using tuangou to reduce IP transit costs", IEEE/ACM Transactions on Networking (ToN), 22(5), pp. 1415-1428, 2014 
(1)

ton

\begin{abstract}
Onkar Bhardwaj bio
\[ \begin{array}{l}\text { Onkar Bhardwaj is a graduate student pursuing Ph.D. with the Department of Electrical, } \\ \text { Systems Engineering at Rensselaer Polytechnic Institute, USA. His interests are Commun } \\ \text { Networks, Applications of algorithms and game theory to networks, Parallel Computing. }\end{array} \]
Onkar Bhardwaj bio
\[ \begin{array}{l}\text { Onkar Bhardwaj is a graduate student pursuing Ph.D. with the Department of Electrical, Computer and } \\ \text { Systems Engineering at Rensselaer Polytechnic Institute, USA. His interests are Communication } \\ \text { Networks, Applications of algorithms and game theory to networks, Parallel Computing. }\end{array} \]

Onkar Bhardwaj bio
\[ \begin{array}{l}\text { Onkar Bhardwaj is a graduate student pursuing Ph.D. with the Department of Electrical, Computer and } \\ \text { Systems Engineering at Rensselaer Polytechnic Institute, USA. His interests are Communication } \\ \text { Networks, Applications of algorithms and game theory to networks, Parallel Computing. }\end{array} \]

Onkar Bhardwaj bio
\[ \begin{array}{l}\text { Onkar Bhardwaj is a graduate student pursuing Ph.D. with the Department of Electrical, Computer and } \\ \text { Systems Engineering at Rensselaer Polytechnic Institute, USA. His interests are Communication } \\ \text { Networks, Applications of algorithms and game theory to networks, Parallel Computing. }\end{array} \]

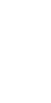

(1)

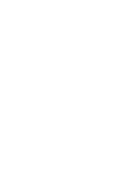


Elliot Anshelevich received the Ph.D. degree in computer science (CS) from Cornell University, Ithaca, NY, USA, in 2005. He had a post-doctoral position with Princeton University, Princeton, NJ, USA, paid for by an NSF Mathematical Sciences Postdoctoral Research Fellowship. He has been a faculty member of the CS Department, Rensselaer Polytechnic Institute (RPI), Troy, NY, USA, since 2006. His research interests include network design problems, algorithmic game theory, local and decentralized routing algorithms, approximation algorithms, and information propagation in both social and computer networks. He is interested in essentially all algorithms for large decentralized networks, including networks with strategic agents. Dr. Anshelevich received an Outstanding Early Research Award from the School of Science, RPI, in 2011. 
Koushik Kar received the B.Tech. degree in electrical engineering from the Indian Institute of Technology, Kanpur, India, in 1997, and the M.S. and Ph.D. degrees in electrical and computer engineering from the University of Maryland, College Park, MD, USA, in 1999 and 2002, respectively. He is currently an Associate Professor with the Electrical, Computer, and Systems Engineering Department, Rensselaer Polytechnic Institute, Troy, NY, USA. His primary research interests are in modeling, performance optimization, and protocol development for communication networks. His recent research work is pri- marily focused on scheduling/access control, traffic engineering, congestion control, and energy management issues in the Internet as well as wireless networks. Dr. Kar is currently an Associate Editor of the IEEE Transactions on Mobile Computing. He received the Career Award from the National Science Foundation in 2005. 


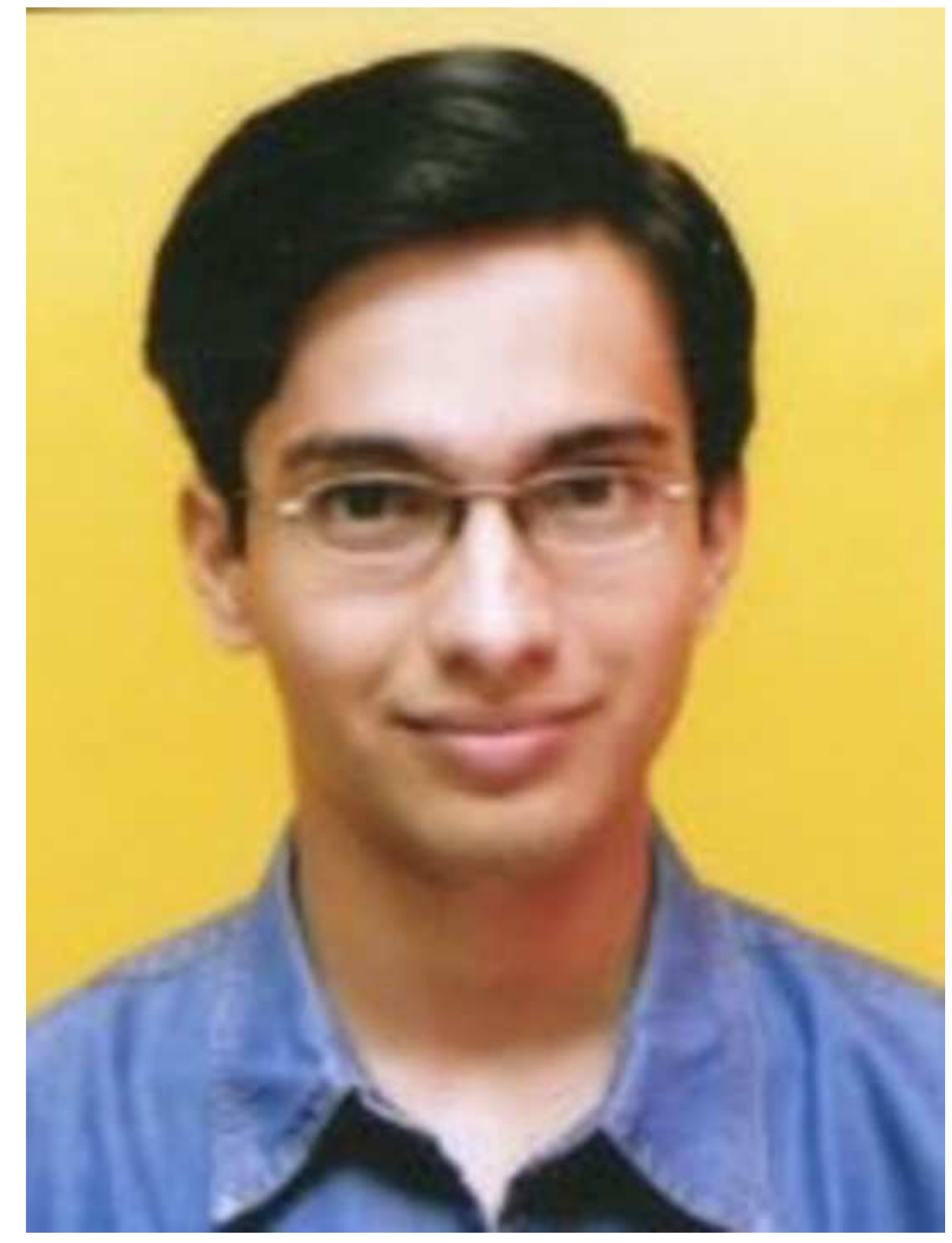

\section{Onkar Bhardwaj photo}

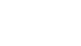
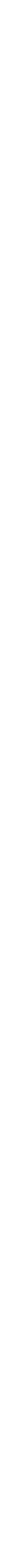


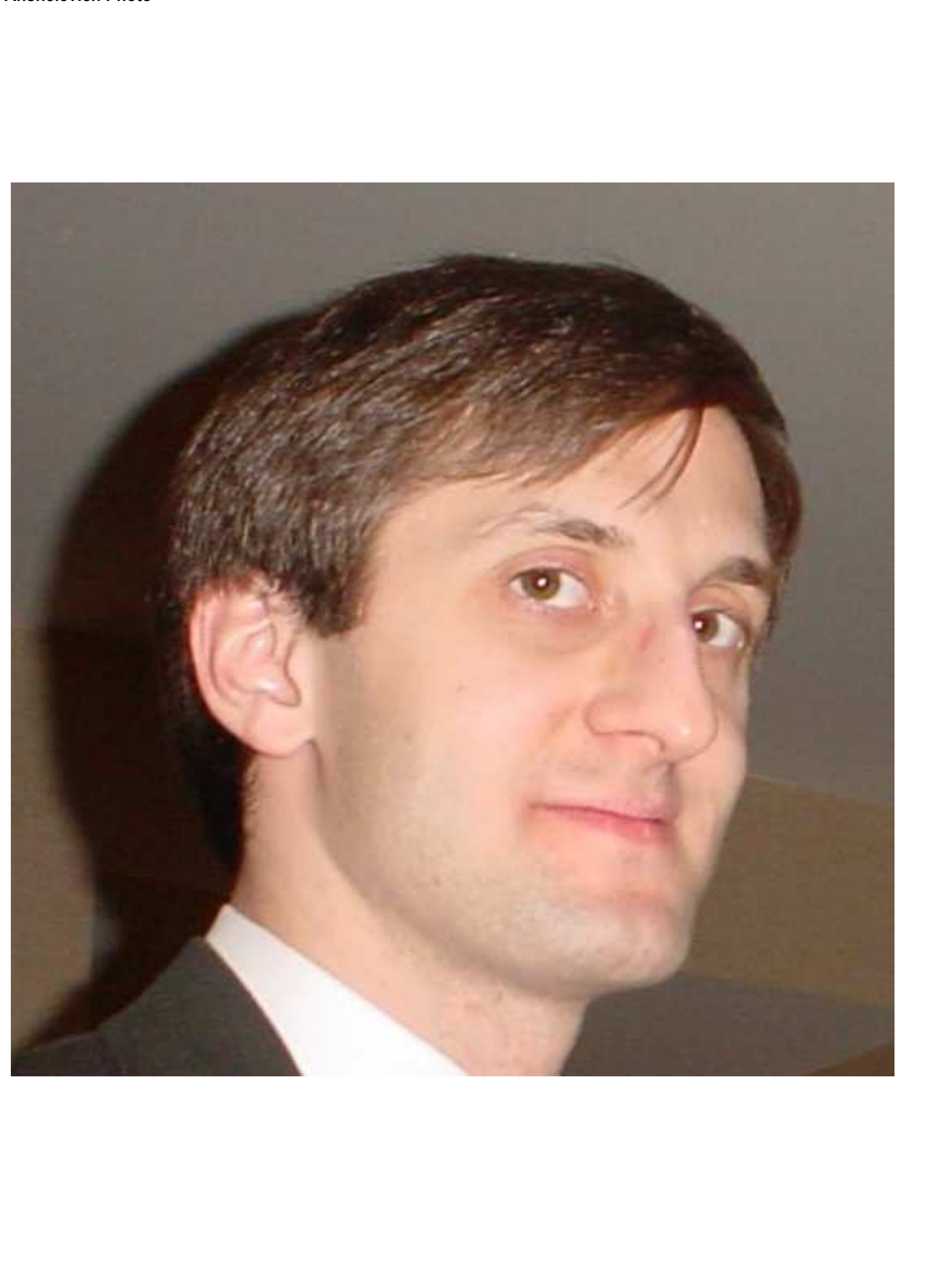

\section{Elliot Anshelevich Photo}

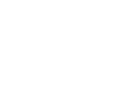

$\sqrt{2}+x^{2}$

(n)
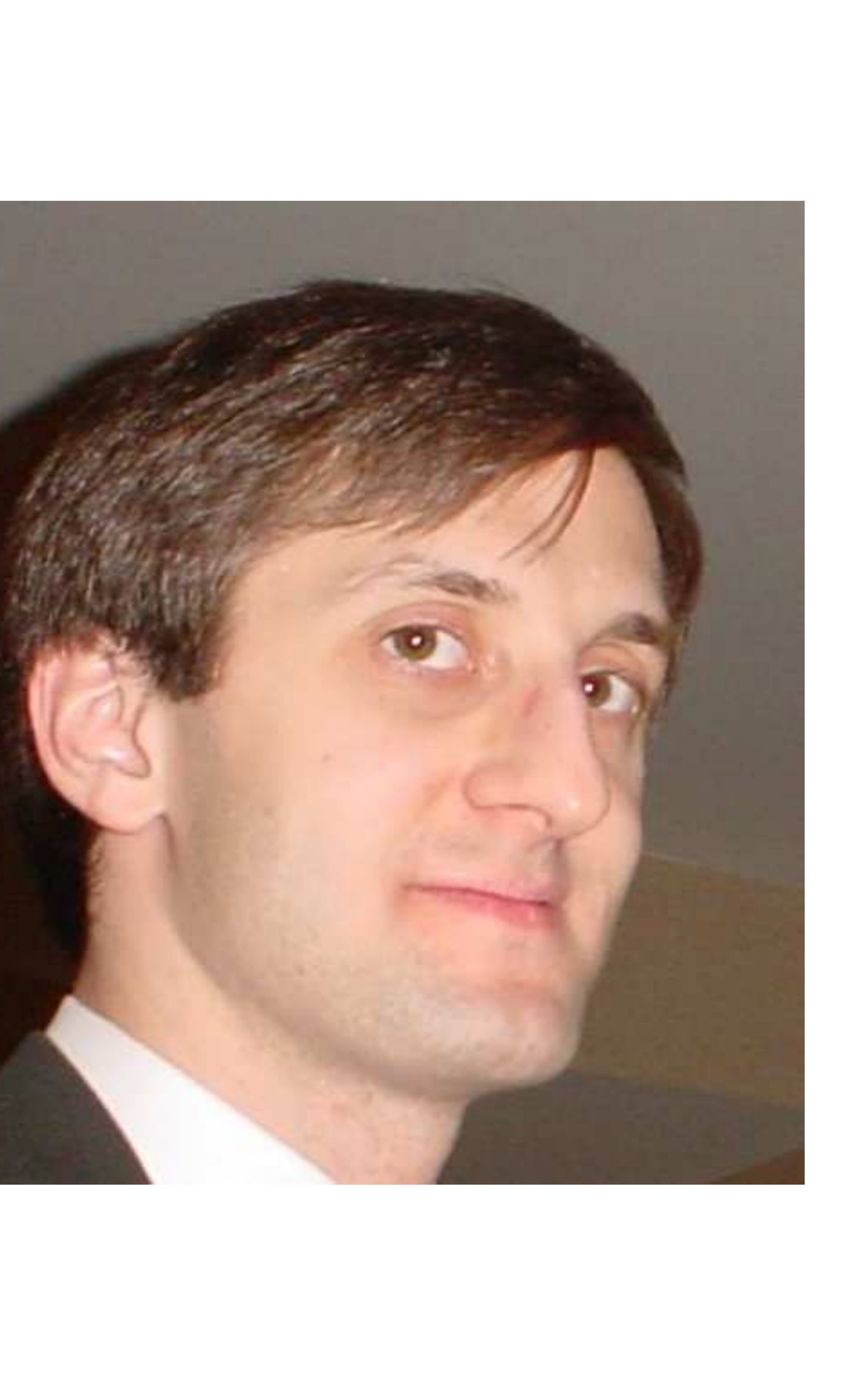
